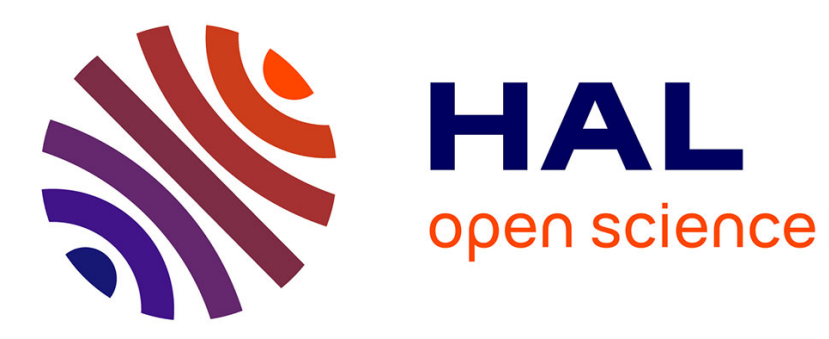

\title{
Linearly Repetitive Delone Sets
}

José Aliste-Prieto, Daniel Coronel, Maria Isabel Cortez, Fabien Durand, Samuel Petite

\section{To cite this version:}

José Aliste-Prieto, Daniel Coronel, Maria Isabel Cortez, Fabien Durand, Samuel Petite. Linearly Repetitive Delone Sets. Mathematics of Aperiodic Order, 37, pp.195-222, 2015, 10.1007/978-3-03480903-0_6. hal-02463104

\section{HAL Id: hal-02463104 https://hal.science/hal-02463104}

Submitted on 31 Jan 2020

HAL is a multi-disciplinary open access archive for the deposit and dissemination of scientific research documents, whether they are published or not. The documents may come from teaching and research institutions in France or abroad, or from public or private research centers.
L'archive ouverte pluridisciplinaire HAL, est destinée au dépôt et à la diffusion de documents scientifiques de niveau recherche, publiés ou non, émanant des établissements d'enseignement et de recherche français ou étrangers, des laboratoires publics ou privés. 


\title{
Linearly repetitive Delone sets
}

\author{
José Aliste-Prieto, Daniel Coronel, María Isabel Cortez, Fabien \\ Durand and Samuel Petite
}

Mathematics Subject Classification (2010). 37B50,

Keywords. Delone sets, tiling systems, linearly repetitive, Voronoï cell.

\section{History and motivations}

The notion of linearly recurrent subshift has been introduced in Du, DHS to study the relations between the substitutive dynamical systems and the stationary dimension groups. In an independent way, the similar notion of linearly repetitive Delone sets of the Euclidean space $\mathbb{R}^{d}$ appears in LP1. For a Delone set $X$ of $\mathbb{R}^{d}$, the repetitivity function $M_{X}(R)$ is the least $M$ (possibly infinite) such that every closed ball $B$ of radius $M$ intersected with $X$ contains a translated copy of any patch with diameter smaller than $2 R$.

A Delone set $X$ is said linearly repetitive if there exists a constant $L$ such that $M_{X}(R)<L R$ for all $R>0$. Observe that we can assume that the constant $L$ is greater than 1 . According to the following theorem, the slowest growth for the repetitivity function of an aperiodic Delone set is linear.

Theorem 1 ([LP1] Theo. 2.3). Let $d \geq 1$. There exists a constant $c(d)>0$ such that for any Delone set $X$ of $\mathbb{R}^{d}$ such that

$$
M_{X}(R)<c(d) R \quad \text { for some } R>0,
$$

then $X$ has a non-zero period.

Even more, if for some $R, M_{X}(R)<\frac{4}{3} R$, then the Delone set $X$ is a crystal i.e. has $d$ independent periods (Theo. 2.2 [LP1] )

The classical examples of aperiodic Delone systems (i.e. arising form substitution) are linearly repetitive.

Lemma 2 ([S02 Lem. 2.3). A primitive self similar tiling is linearly repetitive.

In many senses that we will not specify, the family of linearly repetitive Delone sets is small inside the family of all the Delone sets of the Euclidean 
space $\mathbb{R}^{d}$. For instance, in the class of Sturmian subshifts, several authors MH, Du1, Du, LP2] show the following result.

Proposition 3. The Sturmian subshift associated to an irrational number $\alpha$ is linearly recurrent if and only if the coefficients of the continued fraction of $\alpha$ are bounded.

Let us recall that for the standard topology, the set of numbers with bounded continued fraction are badly approximable by rational numbers. It is known that they form a Baire meager set, with 0 Lebesgue measure but with Hausdorff dimension 1.

As we shall see, the linearly repetitive Delone sets possess many rigid properties. In the next section we present some combinatorial properties of these sets. For instance, their complexity appears to be the slowest possible among all the aperiodic repetitive Delone sets. Section 3 is devoted to the structure of the hull of an aperiodic linearly repetitive Delone set. A tower system with uniform bound is described. We deduce from this the main properties of the system. We focus in Section 4 on the ergodic properties of dynamical systems associated to linearly repetitive Delone sets. They are stricly ergodic (i.e. each patch appears with a frequency). But they are not wild since they are never measurably mixing. They satisfy also a subbaditive ergodic theorem. We present a characterization of the linear repetitivity by using a bound on the frequencies of the occurences of the patches. The dynamical factors of these systems are studied in Section 5. They admit as factors just a finite number of non conjugate aperiodic Delone systems. We give also a characterization of their continuous and measurable eigenvalues by studying cohomological equations. The last section concerns the deformation of linearly repetitive Delone sets: each one is the image through a Lipschitz map of a lattice in $\mathbb{R}^{d}$.

\section{Combinatorial properties}

In this section we give the basic definitions and combinatorial properties concerning linearly repetitive Delone sets of $\mathbb{R}^{d}$. Most of these properties are obviuous for self-similar tilings. In the rest of this paper we suppose that all the Delone sets have finite local complexity. We denote by $B_{R}(x)$ the Euclidean closed ball of radius $R>0$ centred at the point $x \in \mathbb{R}^{d}$.

\subsection{Return vectors to a patch}

Let $X$ be a Delone set having finite local complexity. Let us denote $\left(r_{X}, R_{X}\right)$ the constants of discretness and relative density associated to $X$. A $R$-patch is a set of the kind $\mathrm{P}=X \cap B_{R}(x)$ centred at some point $x \in X$ and for some $R>R_{X}{ }^{1}$. For a $R$-patch $\mathrm{P}$, we define the set

$$
\mathcal{R}_{\mathrm{P}}(X)=\left\{v \in \mathbb{R}^{d}: \mathrm{P}+v \text { is a } R \text {-patch of } X\right\} .
$$

\footnotetext{
${ }^{1}$ note: a given patch may be defined by several centers $x$ and radius $R$. So when we consider a $R$-patch $\mathrm{P}$, we choose a center $x_{\mathrm{P}}$ and a radius $R$.
} 
It is called the set of return vectors to $\mathrm{P}$. For a fixed center $x_{\mathrm{P}}$ of $\mathrm{P}$, any point in $\mathcal{R}_{\mathrm{P}}(X)+x_{\mathrm{P}}=: X_{\mathrm{P}}$ is an occurence of the patch $\mathrm{P}$.

Observe that the null vector 0 always belongs to $\mathcal{R}_{\mathrm{P}}(X)$. It is straightforward to check that $X_{\mathrm{P}}$ is a Delone set when $X$ is repetitive. Furthermore, $X_{\mathrm{P}}$ has finite local complexity because $X_{\mathrm{P}}-X_{\mathrm{P}} \subset X-X$.

When $X$ is aperiodic and linearly repetitive with constant $L$, there are uniform bounds on the constants $r_{X_{\mathrm{P}}}$ and $R_{X_{\mathrm{P}}}$ associated to the Delone set $X_{\mathrm{P}}$. The following lemma shows that two occurrences of a patch can not be too close. The proof can be found in [Le Lem. 2.1 and in So2, Du1].

Lemma 4. Let $X$ be a linearly repetitive aperiodic Delone set with constant $L>1$. Then, for every patch $P=X \cap B_{R}(x)$ with $x \in X, R>0$, we have

$$
\frac{R}{L+1} \leq r_{X_{P}} \leq R_{X_{P}} \leq L R .
$$

Proof. By contradiction: let us assume there exist $x \neq y \in X$ with

$$
\left(X \cap B_{R}(x)\right)-x=X \cap B_{R}(y)-y
$$

and

$$
r_{X} \leq\|x-y\|<\frac{R}{(L+1)} .
$$

Then for any point $z^{\prime}$ in $B_{R}(x) \cap X$, we have $z^{\prime}+(y-x) \in X$. For any $z \in X$, the set $X \cap B_{R}(x)$ contains a translated copy centered in $z^{\prime} \in X \cap B_{R}(x)$ of the patch $B_{\frac{R}{L+1}}(z) \cap X$. Thus $z^{\prime}+(y-x) \in X \cap B_{\frac{R}{L+1}}\left(z^{\prime}\right)$ and finally $z+(y-x) \in X$ and so $X+(y-x) \subset X$. In a similar way we obtain $X+(x-y) \subset X$, so that finally we get $X+x-y=X$ contradicting the aperiodicity of $X$.

This repulsion property on the occurrences of patches has several consequences on the combinatorics of the Delone set $X$.

First of all on the complexity. Let us denote $N_{X}(R)$ the number of different $R$-patches $B_{R}(x) \cap X$ with $x \in X$, up to translation. Since any ball of radius $M_{X}(R)$ contains the centers of occurencies of any $R$-patch, we easily deduce that $N_{X}(R)^{\frac{1}{d}}=O\left(M_{X}(R)\right)$ as $R \rightarrow \infty($ see [LP2] $)$.

Lemma 5 ([L], Lem. 2.2). Let $X$ be an aperiodic linearly repetitive Delone set. Then

$$
\liminf _{R \rightarrow+\infty} \frac{N_{X}(R)}{R^{d}}>0 .
$$

From this, we conclude that for an aperiodic linearly repeptitive Delone set $M_{X}(R)=O\left(N_{X}(R)^{\frac{1}{d}}\right)$ as $R \rightarrow \infty$.

Proof. As $X$ is relatively dense, there exist constants $\lambda_{1}>0$ and $R_{1}>0$ such that

$$
\sharp\left(X \cap B_{R}(x)\right) \geq \lambda_{1} R^{d} \quad \text { for any } x \in X, R \geq R_{1} \text {. }
$$


By the previous lemma all the patches $(X-x) \cap B_{R}(0)$ for $x \in X \cap B_{\frac{R}{3(L+1)}}(0)$ are pairwise different. Thus for any $R \geq 3(L+1) R_{1}$, we have

$$
N_{X}(R) \geq \sharp\left(X \cap B_{\frac{R}{3(L+1)}}(0)\right) \geq \lambda_{1}\left(\frac{R}{3(L+1)}\right)^{d},
$$

that gives us the result.

Another property is on the hierarchical structure of the linearly repetitive Delone sets, that is quite simple: for any size $R>0$, it is possible to decompose the Delone set into big patches (each one containing a $R$-patch), so that the number of these patches, up to translations, is independent of the size $R$. To be more precise, we need the notion of Voronoï cell of a patch. For a $\left(r_{X}, R_{X}\right)$-Delone set $X$, the Voronoï cell $V_{x}$ of a point $x \in X$ is the set

$$
V_{x}=\left\{y \in \mathbb{R}^{d}:\|y-x\| \leq\left\|y-x^{\prime}\right\|, \forall x^{\prime} \in X\right\} .
$$

It is then direct to check that any Voronoï cell $V_{x}$ is a convex polyhedra, its diameter is smaller or equal to $2 R_{X}$ and it contains the ball $B_{\frac{r_{X}}{2}}(x)$. Moreover when the Delone set $X$ is of finite local complexity, the collection of Voronoï cells $\left\{V_{x}\right\}_{x \in X}$ forms a tiling of $\mathbb{R}^{d}$ of finite local complexity.

For a patch $R$-patch $\mathrm{P}=B_{R}\left(x_{\mathrm{P}}\right) \cap X$ of a repetitive Delone set $X$, we denote by $V_{\mathrm{P}, x}$ the Voronoï cell associated to the Delone set $X_{\mathrm{P}}$ and an occurence $x \in X_{\mathrm{P}}$. Notice that the Voronoï cell associated to the set of return vectors $\mathcal{R}_{\mathrm{P}}(X)$ and a return vector $v \in \mathcal{R}_{\mathrm{P}}(X)$, is the Voronoï cell of the occurence $x_{\mathrm{P}}+v \in X_{\mathrm{P}}$ translated by the vector $-x_{\mathrm{P}}$.

It follows by Lemma 4 that for an aperiodic linearly repetitive Delone set with constant $L$, for any $R$-patch $\mathrm{P}$,

$$
\operatorname{diam} V_{\mathrm{P}, x} \leq 2 L R, \quad B_{\frac{R}{2(L+1)}}(x) \subset V_{\mathrm{P}, x}, \quad \text { for any } x \in X_{\mathrm{P}} .
$$

Lemma 6 (CDP Lem. 11). Let $X$ be an aperiodic linearly repetitive Delone set with constant $L$. There exists an explicit positive constant $c(L)$ such that for every $R>0$ and every $R$-patch $P=X \cap B_{R}(x)$, the collection $\left\{X \cap V_{P, x}\right.$ : $\left.x \in X_{P}\right\}$ contains at most $c(L)$ elements up to translation.

Observe here that the bound, explicit in the proof, does not depend on the combinatorics of $X$ but just on the constant of repetitivity.

Proof. Let us consider $B$ the union of Voronoï cells $V_{\mathrm{P}, x}, x \in X_{\mathrm{P}}$ that intersects the ball $B_{L^{2} R}(0)$. We have then

$$
B_{L^{2} R}(0) \subset B \subset B_{L^{2} R+2 L R}(0) .
$$

By linear repetitivity, $B \cap X$ contains a translated copy of any patch of the kind $X \cap V_{\mathrm{P}, x}$ with $x \in X_{\mathrm{P}}$. Since any Voronoï cell contains a ball of radius $\frac{R}{2(L+1)}$, the number of patches in $B \cap X$ of the kind $X \cap V_{\mathrm{P}, x}$ with $x \in X_{\mathrm{P}}$ is smaller than

$$
\frac{\operatorname{vol} B_{R L(L+2)}(0)}{\operatorname{vol} B_{\frac{R}{2(L+1)}}(0)} \leq\left(2 L(L+2)^{2}\right)^{d}=c(L) .
$$


Even stronger, the next lemma gives for an aperiodic linearly repetitive Delone set, an uniform bound (in $R$ ) on the number of occurrences of a patch inside a ball of radius $K R$.

Lemma 7. Let $X$ be an aperiodic linearly repetitive Delone set with constant $L \geq 1$, and let $K \geq L$. Then for any $R$-patch $P$ of $X$ and any point $y \in \mathbb{R}^{d}$,

$$
\sharp\left\{v \in \mathbb{R}^{d} ; P-v \subset B_{K R}(y) \cap X\right\} \leq 12^{d} K^{d} L^{d} .
$$

Proof. Let $B$ be the union of all the Voronoï cells $V_{\mathrm{P}, x}, x \in X_{\mathrm{P}}$ that intersect the ball $B_{K R}(y)$. It follows that

$$
B \subset B_{K R+2 L R}(y) .
$$

By Lemma 4. the sets $B_{\frac{R}{2(L+1)}}(z)$, where the points $z \in B_{K R}(y) \cap X_{\mathrm{P}}$ are occurrences of $\mathrm{P}$, are pairwise disjoint and are included in $B$. Then it follows that

$$
\sharp\left\{v \in \mathbb{R}^{d} ; \mathrm{P}-v \subset B\right\} \leq \frac{\operatorname{vol}(B)}{\operatorname{vol} B \frac{R}{2(L+1)}(0)} \leq 2^{d}(K+2 L)^{d}(L+1)^{d},
$$

that gives us the result.

Here again, observe that the bound depends just on the repetitivity constant.

\section{Structure of the hull of a linearly repetitive Delone set}

\subsection{Background on Solenoids, boxes}

In this section, we will see the specific geometrical structure of the associated hull $\Omega$ of an aperiodic repetitive Delone set. We recall here, from [BBG, BG], the local structure of this space.

3.1.1. Local transversals and return vectors. Let $\left(\Omega, \mathbb{R}^{d}\right)$ be an aperiodic minimal Delone system. The canonical transversal of $\Omega$ is the set $\Omega^{0}$ composed of all Delone sets in $\Omega$ that contain the origin 0 . This terminology is motivated by the fact that if $Y$ is in $\Omega^{0}$, then every small translation of $Y$ will not be in $\Omega^{0}$. A cylinder in $\Omega$ is a set of the form

$$
C_{Y, S}:=\left\{Z \in \Omega \mid Z \cap B_{S}(0)=Y \cap B_{S}(0)\right\},
$$

where $Y \in \Omega$ and $S>0$ are such that $Y \cap B_{S}(0) \neq \emptyset$. The next lemma is well-known.

Proposition 8. Every cylinder in $\Omega$ is a Cantor set. Moreover, a basis for the topology of $\Omega$ is given by sets of the form

$$
\left\{Z-v \mid Z \in C_{Y, S}, v \in B_{\varepsilon}(0)\right\} .
$$

In particular, the canonical transversal $\Omega^{0}$ is a Cantor set. 
A local transversal in $\Omega$ is a clopen (both closed and open) subset of some cylinder in $\Omega$. By Proposition 8 a local transversal $C$ is a Cantor set. This implies that the recognition radius defined as

$$
\operatorname{rec}(C):=\inf \left\{S>0 \mid C_{Y, S} \subseteq C \text { for all } Y \in C\right\}
$$

is finite. The motivation to define $\operatorname{rec}(C)$ is the following: suppose that a Delone set $Y \in \Omega$ is given and we want to check if $Y$ belongs to $C$. Then it suffices to look whether the patch $Y \cap \bar{B}_{\text {rec }(C)}(0)$ is equivalent to $Y_{i} \cap \bar{B}_{\operatorname{rec}(C)}(0)$ for some $Y_{i}$. Of course, if $C=C_{Y, S}$, then its recognition radius is smaller than $S$. Proposition 8 implies also that the collection

$$
\left\{C_{Y, S} \mid Y \in C, S>\operatorname{rec}(C)\right\}
$$

forms a basis for its topology. Indeed, since $C$ is a Cantor set, it is easy to find a finite set $\left\{Y_{1}, \ldots, Y_{m}\right\}$ in $C$ such that

$$
C=\bigcup_{i=1}^{m} C_{Y_{i}, \operatorname{rec}(C)} .
$$

Given a local transversal $C$ and $D \subseteq \mathbb{R}^{d}$, the following notation will be used throughout the paper:

$$
C[D]=\{Y-x \mid Y \in C, x \in D\} .
$$

As we define a return vector to a patch, one can define the set of return vectors to a local transversal. Given a local transversal $C$ and a Delone set $Y \in \Omega$, we define

$$
\mathcal{R}_{C}(Y)=\left\{x \in \mathbb{R}^{d} \mid Y-x \in C\right\} .
$$

When $Y$ belongs to $C$, we refer to $\mathcal{R}_{C}(Y)$ as the set of return vectors of $Y$ to $C$. The following lemma is standard (see e.g. [C]

Lemma 9. Let $C$ be a local transversal. Then for each $Y \in C$, the set of return vectors $\mathcal{R}_{C}(Y)$ is a repetitive Delone set. Moreover, the following quantities

$$
\begin{aligned}
r(C) & =\frac{1}{2} \inf \left\{\|x-y\| x, y \in \mathcal{R}_{C}(Y), x \neq y\right\}, \quad \text { and } \\
R(C) & =\inf \left\{R>0 \mathcal{R}_{C}(Y) \cap \bar{B}_{R}(y) \neq \emptyset \text { for all } y \in \mathbb{R}^{d}\right\},
\end{aligned}
$$

do not depend on the choice of $Y$ in $C$.

3.1.2. Solenoids and boxes. In this section, we recall some definitions and results of $[\mathrm{BBG}, \mathrm{BG}$ that will be used throughout the paper. The hull $\Omega$ is locally homeomorphic to the product of a Cantor set and $\mathbb{R}^{d}$ (see chapter toolbox). Moreover, there exists an open cover $\left\{U_{i}\right\}_{i=1}^{n}$ of $\Omega$ such that for each $i \in\{1, \ldots, n\}$, there are $Y_{i} \in \Omega, S_{i}>0$ and open sets $D_{i} \subseteq \mathbb{R}^{d}$ such that $U_{i}=C_{Y_{i}, S_{i}}\left[D_{i}\right]$ and the map $h_{i}: D_{i} \times C_{i} \rightarrow U_{i}$ defined by $h_{i}(t, Z)=Z-t$ is a homeomorphism. Furthermore, there are vectors $v_{i, j} \in \mathbb{R}^{d}$ (depending only on $i$ and $j$ ) such that the transition maps $h_{i}^{-1} \circ h_{j}$ satisfy

$$
h_{i}^{-1} \circ h_{j}(t, Z)=\left(t-v_{i, j}, Z-v_{i, j}\right)
$$

at all points $(t, Z)$ where the composition is defined. Following BG, we call such a cover a $\mathbb{R}^{d}$-solenoid's atlas. It induces, among others structures, a 
laminated structure as follows. First, slices are defined as sets of the form $h_{i}\left(D_{i} \times\{Z\}\right)$. Equation (3.3) implies that slices are mapped onto slices. Thus, the leaves of $\Omega$ are defined as the smallest connected subsets that contain all the slices they intersect. It is not difficult to check, using $(3.3)$, that the leaves coincide with the orbits of $\Omega$.

A box in $\Omega$ is a set of the form $B:=C[D]$ where $C$ is a local transversal in $\Omega$, and $D \subseteq \mathbb{R}^{d}$ is an open set such that the map from $D \times C$ to $B$ given by $(x, Y) \mapsto Y-x$ is a homeomorphism. This is true, for instance, if $D \subseteq B_{r(C)}(0)(c f .(3.1))$.

\subsection{Tower systems}

In this section we review the concepts of box decompositions and tower systems introduced in $[\mathrm{BBG}, \mathrm{BG}$. We focus on linearly repetitive Delone sets. The main results of this section can be found in $\mathrm{AC}$. For all this section, $\Omega$ denotes the hull of an aperiodic repetitive Delone set $X$.

3.2.1. Box decompositions and derived tilings. A box decomposition is a finite and pairwise-disjoint collection of boxes $\mathcal{B}=\left\{B_{1}, \ldots, B_{t}\right\}$ in $\Omega$ such that the closures of the boxes in $\mathcal{B}$ cover the hull. For simplicity, we always write $B_{i}=$ $C_{i}\left[D_{i}\right]$, where $C_{i}$ and $D_{i}$ are fixed and $C_{i}$ is contained in $B_{i}$. In particular, the set $D_{i}$ contains 0 . We refer to $C_{i}$ as the base of $B_{i}$. In this way, we call the union of all $C_{i}$ the base of $\mathcal{B}$. The reasoning for fixing a local transversal in each $B_{i}$ comes from the fact that box decompositions can be constructed in a canonical way starting from the set $\mathcal{R}_{C}(Y)$ of return vectors to a given local transversal $C$ (see details in Section chaptre toolbox).

An alternative way of understanding a box decomposition is given by a family of tilings, known as derived tilings, which are constructed by intersecting the box decomposition with the orbit of each Delone set in the hull.

Let us start by recalling some basic definitions about tilings. A tile $T$ in $\mathbb{R}^{d}$ is a compact set that is the closure of its interior (not necessarily connected). A tiling $\mathcal{T}$ of $\mathbb{R}^{d}$ is a countable collection of tiles that cover $\mathbb{R}^{d}$ and have pairwise disjoint interiors. Tiles can be decorated: they may have a color and/or be punctured at an interior point. Formally, this means that decorated tiles are tuples $(T, i, x)$, where $T$ is a tile, $i$ lies in a finite set of colors, and $x$ belongs to the interior of $T$. Two tiles have the same type if they differ by a translation. If the tiles are punctured, then the translation must also send one puncture to the other, and when they are colored, they must have the same color.

To construct a derived tiling, the idea is to read the intersection of the boxes in the box decomposition with the orbit of a fixed Delone set in the hull. In the sequel, it will be convenient to make the following construction. Let $\left\{C_{i}\right\}_{i=1}^{t}$ be a collection of local transversals and $\left\{D_{i}\right\}_{i=1}^{t}$ be a collection of bounded open subsets of $\mathbb{R}^{d}$ containing 0 . Define $\mathcal{B}=\left\{C_{i}\left[D_{i}\right]\right\}_{i=1}^{t}$ and observe that the sets in $\mathcal{B}$ are not necessarily boxes of $\Omega$. For each $Y \in \Omega$, 
define the (decorated) derived collection of $\mathcal{B}$ at $Y$ by

$$
\mathcal{T}_{\mathcal{B}}(Y):=\left\{\left(\overline{D_{i}}+v, i, v\right) \mid i \in\{1, \ldots, t\}, v \in \mathcal{R}_{C_{i}}(Y)\right\} .
$$

The following lemma gives the relation between box decomposition and tilings.

Lemma 10 (Lem. $3.1[\mathrm{AC}]$ ). Let $\mathcal{B}=\left\{C_{i}\left[D_{i}\right]\right\}_{i=1}^{t}$, where the $C_{i}$ 's are local transversals and the $D_{i}$ 's are open bounded subsets of $\mathbb{R}^{d}$ that contain 0 . Then, $\mathcal{B}$ is a box decomposition if and only if $\mathcal{T}_{\mathcal{B}}(Y)$ is a tiling of $\mathbb{R}^{d}$ for every $Y \in \Omega$. In this case, we call $\mathcal{T}_{\mathcal{B}}(Y)$ the derived tiling of $\mathcal{B}$ at $Y$.

Proof. It is easy to see that if $\mathcal{B}$ is a box decomposition, then $\mathcal{T}_{\mathcal{B}}(Y)$ is a tiling for every $Y \in \Omega$. We now show the converse. For convenience, set $C=\cup_{i} C_{i}$. Fix $Y \in \Omega$ and suppose there are $i, j \in\{1, \ldots, t\}, Y_{1} \in C_{i}, Y_{2} \in C_{j}, x_{1} \in D_{i}$ and $x_{2} \in D_{j}$ such that $Y=Y_{1}-x_{1}=Y_{2}-x_{2}$. This implies that the tiles $\overline{D_{i}}-x_{1}$ and $\overline{D_{j}}-x_{2}$ of $\mathcal{T}_{\mathcal{B}}(Y)$ meet an interior point. Since $\mathcal{T}_{\mathcal{B}}(Y)$ is a tiling, these tiles must coincide, and hence $i=j$ and $x_{1}=x_{2}$. We conclude i the maps $h_{i}: C_{i} \times D_{i} \rightarrow C_{i}\left[D_{i}\right]$ given by $(Y, t) \mapsto Y-t$ are one-to-one, and moreover their image are pairwise disjoint. It is then straightforward to check that the maps $h_{i}$ are homeomorphims.

3.2.2. Properly nested box decompositions. A box decomposition $\mathcal{B}^{\prime}=\left\{C_{i}^{\prime}\left[D_{i}^{\prime}\right]\right\}_{i=1}^{t^{\prime}}$ is zoomed out of another box decomposition $\mathcal{B}=\left\{C_{j}\left[D_{j}\right]\right\}_{j=1}^{t}$ if the following properties are satisfied:

(Z.1) If $Y \in C_{i}^{\prime}$ is such that $Y-x \in C_{j}-y$ for some $x \in \overline{D_{i}^{\prime}}$ and $y \in \overline{D_{j}}$, then $C_{i}^{\prime}-x \subseteq C_{j}-y$.

(Z.2) If $x \in \partial D_{i}^{\prime}$, then there exist $j$ and $y \in \partial D_{j}$ such that $C_{i}^{\prime}-x \subseteq C_{j}-y$.

(Z.3) For every box $B^{\prime}$ in $\mathcal{B}^{\prime}$, there is a box $B$ in $\mathcal{B}$ such that $B \cap B^{\prime} \neq \emptyset$ and $\partial B \cap \partial B^{\prime}=\emptyset$.

For each $i \in\left\{1, \ldots, t^{\prime}\right\}$ and $j \in\{1, \ldots, t\}$ define

$$
O_{i, j}=\left\{x \in D_{i}^{\prime} \mid C_{i}^{\prime}-x \subseteq C_{j}\right\} .
$$

(Z.4) For each $i \in\left\{1, \ldots, t^{\prime}\right\}$ and $j \in\{1, \ldots, t\}$,

$$
\overline{D_{i}^{\prime}}=\bigcup_{j=1}^{t} \bigcup_{x \in O_{i, j}} \overline{D_{j}}+x,
$$

where all the sets in the right-hand side of the equation have pairwise disjoint interiors.

Observe that in the case that $D_{j}$ is connected, then properties (Z.1) and (Z.2) imply (Z.4).

Since we are considering the $C_{i}^{\prime}$ 's and $C_{j}$ 's as the bases of the boxes, we ask the following additional property to be satisfied:

(Z.5) The base of $\mathcal{B}^{\prime}$ is included in the base of $\mathcal{B}$, that is, $\cup_{i} C_{i}^{\prime} \subseteq \cup_{j} C_{j}$.

By (Z.4), we have that the tiling $\mathcal{T}_{\mathcal{B}^{\prime}}(Y)$ is a super-tiling of $\mathcal{T}_{\mathcal{B}}(Y)$ in the sense that each tile $T$ in $\mathcal{T}_{\mathcal{B}^{\prime}}(Y)$ can be decomposed into a finite set of tiles of $\mathcal{T}_{\mathcal{B}}(Y)$. By (Z.3), one of these tiles is included in the interior of $T$. 
Lemma 11. For every $j \in\{1, \ldots, t\}$ we have

$$
C_{j}=\bigcup_{i=1}^{t^{\prime}} \bigcup_{x \in O_{i, j}} C_{i}^{\prime}-x
$$

Proof. By the definition of $O_{i, j}$ and (Z.1), it suffices to show that every $Y \in$ $C_{j}$ belongs to the interior of some box $C_{i}^{\prime}\left[D_{i}^{\prime}\right]$. Suppose not, then $Y \in C_{i}^{\prime}-x$ with $x \in \partial D_{i}^{\prime}$ for some $i$ since $\mathcal{B}^{\prime}$ is a box decomposition. Moreover, by (Z.2) we deduce that $Y$ must be in the boundary of some box $B_{j^{\prime}}$ in $\mathcal{B}$, which gives a contradiction.

\subsection{Tower systems of linearly repetitive Delone system.}

A tower system is a sequence of box decompositions $\left(\mathcal{B}_{n}\right)_{n \in \mathbb{N}}$ such that $\mathcal{B}_{n+1}$ is zoomed out of $\mathcal{B}_{n}$ for all $n \in \mathbb{N}$. An iteration of the construction of zoomed out box decomposition gives the following result.

Theorem $12([\mathrm{BBG}])$. The hull of any aperiodic minimal Delone set possesses a tower system.

We have explained in Section 3.2.1 how to construct a box decomposition and in section 3.2 .2 the notion of zoomed out box decomposition. In this section, we specify the construction of a tower system to the linear repetititve case.

Fot a decreasing sequence $\left(C_{n}\right)_{n \in \mathbb{N}}$ of local transversals with diameter going to 0 , and a tower system $\left(\mathcal{B}_{n}\right)_{n}$, we say that $\left(\mathcal{B}_{n}\right)_{n}$ is adapted to $\left(C_{n}\right)_{n}$, if for any $n \in \mathbb{N}$ we have $\mathcal{B}_{n}=\left\{C_{n, i}\left[D_{n, i}\right]\right\}_{i=1}^{t_{n}}$ such that $C_{n}=\cup_{i} C_{n, i}$ and $t_{n}$ is a positive integer. In this case, for each $n \in \mathbb{N}^{*}$ we define, as in (3.4),

$$
O_{i, j}^{(n)}=\left\{x \in D_{n, i} \mid C_{n, i}-x \subseteq C_{n-1, j}\right\}
$$

and

$$
m_{i, j}^{(n)}=\sharp O_{i, j}^{(n)}
$$

for every $i \in\left\{1, \ldots, t_{n}\right\}$ and $j \in\left\{1, \ldots, t_{n-1}\right\}$. The transition matrix of level $n$ (associated to the tower system $\left(\mathcal{B}_{n}\right)_{n \in \mathbb{N}}$ ) is then defined as the matrix $M_{n}=\left(m_{i, j}^{(n)}\right)_{i, j}$, so $M_{n}$ has size $t_{n} \times t_{n-1}$. From (Z.4), we get

$$
\operatorname{vol}\left(D_{n, i}\right)=\sum_{j=1}^{t_{n-1}} m_{i, j}^{(n)} \operatorname{vol}\left(D_{n-1, j}\right) .
$$

Given a box decomposition $\mathcal{B}=\left\{C_{i}\left[D_{i}\right]\right\}_{i=1}^{t}$, define its external and internal radius by

$$
\begin{aligned}
R_{\text {ext }}(\mathcal{B}) & =\max _{i \in\{1, \ldots, t\}} \inf \left\{R>0: B_{R}(0) \supseteq D_{i}\right\} ; \\
r_{\text {int }}(\mathcal{B}) & =\min _{i \in\{1, \ldots, t\}} \sup \left\{r>0: B_{r}(0) \subseteq D_{i}\right\},
\end{aligned}
$$

respectively. Define also $\operatorname{rec}(\mathcal{B})=\max _{i \in\{1, \ldots, t\}} \operatorname{rec}\left(C_{i}\right)$.

With all theses definitions, we can state the following result for aperiodic linearly repetitive Delone systems. 
Theorem 13 (Theo. 3.4 $\mathrm{AC}$ ). Let $X$ be an aperiodic linearly repetitive Delone set with constant $L>1$ and $0 \in X$. Given $K \geq 6 L(L+1)^{2}$ and $s_{0}>0$, set $s_{n}:=K^{n} s_{0}$ and $C_{n}:=C_{X, s_{n}}$ for all $n \in \mathbb{N}$. Then, there exists a tower system $\left(\mathcal{B}_{n}\right)_{n}$ of $\Omega$ adapted to $\left(C_{n}\right)_{n \in \mathbb{N}}$ that satisfies the following additional properties:

i) for every $n \geq 0, C_{n+1} \subseteq C_{n, 1}$;

ii) there exist constants

$$
K_{1}:=\frac{1}{2(L+1)}-\frac{L}{K-1} \quad \text { and } \quad K_{2}:=\frac{L K}{K-1},
$$

which satisfy $0<K_{1}<1<K_{2}$, such that for every $n \in \mathbb{N}$ we have

$$
K_{1} s_{n} \leq r_{\text {int }}\left(\mathcal{B}_{n}\right)<R_{\text {ext }}\left(\mathcal{B}_{n}\right) \leq K_{2} s_{n}
$$

iii) for every $n \in \mathbb{N}$,

$$
\operatorname{rec}\left(\mathcal{B}_{n}\right) \leq(2 L+1) s_{n}
$$

As an application of this result, we have the nice following structure.

Theorem 14. Let $X$ be an aperiodic linearly repetitive Delone set. Then, the tower system of $\Omega$ obtained in Theorem 13 satisfies the following:

1. For every $n \in \mathbb{N}^{*}$, the matrix $M_{n}=\left(m_{i, j}^{(n)}\right)_{i, j}$ has strictly positive coefficients;

2. The matrices $\left\{M_{n}\right\}_{n \in \mathbb{N}^{*}}$ are uniformly bounded in size and norm.

In the self-similar case, the family of matrices $\left\{M_{n}\right\}$ can be reduced to only one element.

Proof. Take the notations of Theorem 13. Indeed, by the definition of linearl repetitivity, we have $M_{X}\left(\operatorname{rec}\left(\mathcal{B}_{n}\right)\right) \leq L \operatorname{rec}\left(\mathcal{B}_{n}\right)$ for all $n \in \mathbb{N}^{*}$. Combining this with $(3.8)$, the left-hand inequality of $(3.7)$ and the definition of $s_{n}$ we get

$$
M_{X}\left(\operatorname{rec}\left(\mathcal{B}_{n}\right)\right) \leq \frac{L(2 L+1)}{K K_{1}} r_{\text {int }}\left(\mathcal{B}_{n+1}\right) .
$$

Since $K \geq 6 L(L+1)^{2}$, it follows that $L(2 L+1) \leq K_{1} K$ and we obtain for all $n \geq 0$

$$
M_{X}\left(\operatorname{rec}\left(\mathcal{B}_{n}\right)\right) \leq r_{\text {int }}\left(\mathcal{B}_{n+1}\right) .
$$

Thus any $\operatorname{rec}\left(\mathcal{B}_{n}\right)$-patch occurs in a set $D_{n+1, i} \cap Y$ for any $Y \in C_{n+1, i}$, and the coefficients $m_{i, j}^{(n)}$ are positive. Moreover, since $D_{n, i}$ is included in a ball of radius $R_{\text {ext }}\left(\mathcal{B}_{n-1}\right)$ and each $D_{n-1, j}$ contains a ball of radius $r_{\text {int }}\left(\mathcal{B}_{n-1}\right)$, we deduce from 3.6 that

$$
\sum_{j=1}^{t_{n-1}} m_{i, j}^{(n)} \leq\left(\frac{R_{\mathrm{ext}}\left(\mathcal{B}_{n-1}\right)}{r_{\mathrm{int}}\left(\mathcal{B}_{n-1}\right)}\right)^{d} \leq\left(K \frac{K_{2}}{K_{1}}\right)^{d} .
$$

So we get that the matrices $\left\{M_{n}\right\}_{n}$ are uniformly bounded. 


\section{Ergodic properties of linearly repetitive system}

\subsection{Background on transverse invariant measure}

A Borel measure $\mu$ on the hull $\Omega$ of a repetitive Delone set is translation invariant if $\mu(B-v)=\mu(B)$ for every Borel set $B$ and $v \in \mathbb{R}^{d}$. It is wellknown that any continuous $\mathbb{R}^{d}$ action on a compact space admits an invariant measure.

Let $C$ be a local transversal and $0<r<r(C)$. Each translation invariant measure $\mu$ induces a measure $\nu$ on $C$ (see Gh for the general construction): given a Borel subset $V$ of $C$, its transverse measure is defined by

$$
\nu(V)=\frac{\mu\left(V\left[B_{r}(0)\right]\right)}{\operatorname{vol}\left(B_{r}(0)\right)},
$$

where vol denotes the Euclidean volume in $\mathcal{R}^{d}$. This gives a measure on each $C$, which does not depend on small $r$. The collection of all measures defined in this way is called the transverse invariant measure induced by $\mu$. It is invariant in the sense that if $V$ is a Borel subset of $C$ and $x \in \mathbb{R}^{d}$ is such that $V-x$ is a Borel subset of another local transversal $C^{\prime}$, then $\nu(V-x)=\nu(V)$. Conversely, the measure $\mu$ of any box $B$ written as $C[D]$ may be computed by the equation

$$
\mu(C[D])=\operatorname{vol}(D) \times \nu(C) .
$$

For a tower system $\left(\mathcal{B}_{n}\right)_{n \geq 0}$ where $\mathcal{B}_{n}=\left\{C_{n, i}\left[D_{n, i]}\right\}_{i=1}^{t_{n}}\right.$ from (Z.4), Lemma 11 and the definition of transverse invariant measures, we get

$$
\nu\left(C_{n-1, j}\right)=\sum_{i=1}^{t_{n}} \nu\left(C_{n, i}\right) m_{i, j}^{(n)} .
$$

Fix $n \in \mathbb{N}$. From the relation $\mu\left(C_{n, i}\left[D_{n, i}\right]\right)=\operatorname{vol}\left(D_{n, i}\right) \nu\left(C_{n, i}\right)$ and the fact that $\mathcal{B}_{n}$ is a box decomposition, it follows that

$$
\sum_{j=1}^{t_{n}} \operatorname{vol}\left(D_{n, j}\right) \nu\left(C_{n, j}\right)=1 \text {. }
$$

\subsection{Unique ergodicity and speed of convergence}

When the system $\left(\Omega, \mathbb{R}^{d}\right)$ has an unique translation invariant probability measure, the system is called uniquely ergodic. The unique ergodicity implies combinatorial properties for the Delone set. The dynamical system $\left(\Omega, \mathbb{R}^{d}\right)$ is uniquely ergodic, if and only if any Delone set $X \in \Omega$ has uniform patch frequencies, i.e., any patch $\mathrm{P}$ occurs with a positive frequency; more precisely: Let $X_{\mathrm{P}}$ be the set of occurences of the patch $\mathrm{P}$ in $X$, and let $\left(D_{N}\right)_{N}$ be a nested sequence of $d$-cube $D_{N}$ of side $N$, then the following limit exists.

$$
\lim _{N \rightarrow \infty} \frac{\sharp X_{\mathrm{P}} \cap D_{N}}{\operatorname{vol}\left(D_{N}\right)}=\text { : freq }(\mathrm{P}) .
$$

The number freq $(\mathrm{P})$ is called the frequency of $\mathrm{P}$. Notice the difference with the standard Birkhoff's ergodic Theorem that asserts a convergence only for almost all Delone set of the hull. 
Theorem 15. Let $X$ be an aperiodic linearly repetitive Delone set of $\mathbb{R}^{d}$ and $\Omega$ its hull. Then the system $\left(\Omega, \mathbb{R}^{d}\right)$ is uniquely ergodic.

The original proof is due to Lagarias and Pleasants in [LP2]. By using the identification between a transverse invariant measure and the inverse limit of top homologies of branched manifolds, the authors in BBG show that in the case described in Theorem 14 the system is uniquely ergodic. This proof is independent of the original one.

Actually for linearly repetitive system, we can be much more precise and give informations on the speed of convergence of the limit. For instance the following is a stronger result of Lagarias and Pleasants [LP2, that implies the unique ergodicity.

Theorem 16 ([LP2]). Let $X$ be a linearly repetitive Delone set of $\mathbb{R}^{d}$. There exists a $\delta>0$ such that, for every patch $P$ of $X$, there is a number freq $(P)$ so that

$$
\left|\frac{X_{P} \cap \operatorname{Dom}_{N}}{\operatorname{vol}\left(\operatorname{Dom}_{N}\right)}-\operatorname{freq}(P)\right|=O\left(N^{-\delta}\right),
$$

where Dom $_{N}$ is either a d-cube with side $N$ or a ball of radius $N$. The $O$ constant may depend on the patch $P$.

In [AC], a proof of this theorem is given using the structure Theorem 14 and relating the constant $\delta$ with the matrices $M_{n}$ by the following way

$$
\delta=d-\log _{K}\left(1-\sup _{n}\left\|M_{n}\right\|_{1}^{-1}\left\|M_{n+1}\right\|_{1}^{-1}\right),
$$

where $\log _{K}$ denotes the logarithm in base $K$.

\subsection{Non-mixing properties}

A translation invariant probability measure $\mu$ on a the hull $\Omega$ of a Delone set is said to be measurably strongly mixing if for any Borel sets $A, B$ in $\Omega$,

$$
\lim _{\|v\| \rightarrow \infty} \mu((A-v) \cap B)=\mu(A) \mu(B) .
$$

In this section, we show the following proposition which is analogous to theorem of Dekking and Keane [DK] for substitutive subshifts.

Proposition 17 ( $\mathrm{C} 0)$. Let $X$ be a linearly repetitive Delone set of $\mathbb{R}^{d}$ and $\Omega$ its hull. Then the system $\left(\Omega, \mathbb{R}^{d}\right)$ is not measurably strongly mixing.

The proof's strategy is the same as for self-similar tiling in [So1] or for linear recurrent Cantor system in [CDHM]. But we need sharp estimates on the transverse measures of clopen sets, provided by Theorem 13 .

Assume that the Delone set $X$ is aperiodic and linearly repetitive with constant $L$. Let $\mu$ be the unique translation invariant probability measure on the hull $\Omega$, and let $\nu$ be the associated transverse invariant measure. Let $\left(\mathcal{B}_{n}\right)_{n \geq 0}$ be the tower system given by Theorem 13 where for each integer $n$, $\mathcal{B}_{n}=\left\{C_{n, i}\left[D_{n, i}\right]\right\}_{i=1}^{t_{n}}$. 
Lemma 18. For the tower system of $\Omega$ given by Theorem 13 , we have

$$
\inf _{\substack{n \geq 1 \\ 1 \leq i \leq n}} \operatorname{vol}\left(D_{n, i}\right) \nu\left(C_{n, i}\right)>\left(\frac{K_{1}}{K K_{2}}\right)^{d}=: c>0 .
$$

Proof. With the equation 4.1, for any $1 \leq i \leq t_{n}$, we get

$$
\nu\left(C_{n, i}\right) \geq \sum_{j=1}^{t_{n+1}} \nu\left(C_{n+1, j}\right)
$$

By definition, for any $1 \leq i \leq t_{n}$, the domain $D_{n, i}$ contains a ball or radius $r_{\text {int }}\left(\mathcal{B}_{n}\right)$ and for $1 \leq j \leq t_{n+1}$ the domain $D_{n+1, j}$ is included in a ball of radius $R_{\text {ext }}\left(\mathcal{B}_{n+1}\right)$. Thus, as in the proof of Theorem 14 , we deduce from Theorem 13

$$
\frac{\operatorname{vol}\left(D_{n+1, j}\right)}{\operatorname{vol}\left(D_{n, i}\right)} \leq\left(\frac{R_{\text {ext }}\left(\mathcal{B}_{n-1}\right)}{r_{\text {int }}\left(\mathcal{B}_{n-1}\right)}\right)^{d} \leq\left(K \frac{K_{2}}{K_{1}}\right)^{d}=c^{-1} .
$$

Thus it follows from 4.2, that for any $n \geq 0$ and $1 \leq i \leq t_{n}$

$$
\operatorname{vol}\left(D_{n, i}\right) \nu\left(C_{n, i}\right) \geq \sum_{j=1}^{t_{n+1}} c \operatorname{vol}\left(D_{n+1, j}\right) \nu\left(C_{n+1, j}\right)=c .
$$

For the tower system $\left(\mathcal{B}_{n}\right)_{n}$, we define as in Definition 3.4 , for integers $p \geq n>0$

$$
O_{i, j}^{(p, n)}:=\left\{x \in D_{p, i} \mid C_{p, i}-x \subseteq C_{n-1, j}\right\}, \text { for } 1 \leq i \leq t_{p} ; 1 \leq j \leq t_{n-1}
$$

and

$$
m_{i, j}^{(p, n)}=\sharp O_{i, j}^{(p, n)} .
$$

Then it is straightforward to check that the $t_{p} \times t_{n-1}$ matrix

$$
\left(m_{i, j}^{(p, n)}\right)_{i, j}=M_{p} \cdots M_{n} .
$$

Lemma 19. For the tower system of $\Omega$ given by Theorem 13 , we have for $n \geq 2$, and $1 \leq j \leq t_{n}$

$$
\lim \inf _{p \rightarrow+\infty} \min _{1 \leq i \leq t_{p}} \frac{m_{i, j}^{(p, n)}}{\operatorname{vol}\left(D_{i, p}\right)} \geq \nu\left(C_{n-1, j}\right)\left(\frac{K_{1}}{K_{2}}\right)^{d} c .
$$

Proof. Let $X \in \cap_{n \geq 0} C(n)$. By the unique ergodicity, we have

$$
\lim _{R \rightarrow+\infty} \frac{1}{\operatorname{vol}\left(B_{R}(0)\right)} \sharp\left\{B_{R}(0) \cap \mathcal{R}_{C_{n, j}}(X)\right\}=\nu\left(C_{n, j}\right) .
$$

Since for every $p>n$, the set $C_{p} \subset C_{p-1,1}$, we get for any $1 \leq i \leq t_{p}$,

$$
m_{i, j}^{(p, n)} \geq m_{1, j}^{(p-1, n)} \geq \sharp\left\{D_{p-1, j} \cap \mathcal{R}_{C_{n-1, j}}(X)\right\} .
$$


Hence we conclude by this inequality and inequality 4.5 that

$\lim \inf _{p \rightarrow+\infty} \min _{1 \leq i \leq t_{p}} \frac{m_{i, j}^{(p, n)}}{\operatorname{vol}\left(D_{p, i}\right)} \geq \lim \inf _{p \rightarrow+\infty} \frac{\sharp\left\{D_{p-1, j} \cap \mathcal{R}_{C_{n-1, j}}(X)\right\}}{\operatorname{vol}\left(D_{p, i}\right)}$

$$
\begin{aligned}
& \geq c \lim _{\inf _{p}} \frac{\sharp\left\{D_{p-1, j} \cap \mathcal{R}_{C_{n-1, j}}(X)\right\}}{\operatorname{vol}\left(D_{p-1, j}\right)} \\
& \geq c \lim _{p} \frac{\sharp\left\{B_{r_{\text {int }}\left(\mathcal{B}_{p-1}\right)}(0) \cap \mathcal{R}_{C_{n-1, j}}(X)\right\}}{\operatorname{vol}\left(B_{\frac{K_{2}}{K_{1}} r_{\text {int }}\left(\mathcal{B}_{p-1}\right)}(0)\right)},
\end{aligned}
$$

since $D_{p-1, j}$ contains the ball $B_{r_{\text {int }}\left(\mathcal{B}_{p-1}\right)}(0)$ and is contained in the ball $B_{R_{\text {ext }}\left(\mathcal{B}_{p-1}\right)}(0) \subset B_{\frac{K_{2}}{K_{1}} r_{\text {int }}\left(\mathcal{B}_{p-1}\right)}(0)$. We obtain the conclusion by the equality 4.7.

Now we are able to prove Proposition 17

Proof of Proposition 17. Let $n$ be an integer such that $\nu\left(C_{n}\right)<\left(\frac{K_{1}}{K_{2}}\right)^{d} c^{2}$. For $p \geq n$, Let $\mathcal{F}_{p, 1} \subset \mathbb{R}^{d}$ be the set of vector $v$ such that there exists a $1 \leq j \leq t_{p}$ satisfying $C_{p, 1}-v \cap C_{p, j} \neq \emptyset$ and $D_{p, j}-v \cap D_{p, 1} \neq \emptyset$. Let $\tilde{C}(n, v)=\left(C_{n, 1}-v\right) \cap C_{n, 1}$. We will show that

$$
\lim \inf _{p \rightarrow \infty} \inf _{v \in \mathcal{F}_{p, 1}} \nu(\tilde{C}(n, v))>\nu\left(C_{n, 1}\right)^{2}
$$

which implies that the system $\left(\Omega, \mathbb{R}^{d}\right)$ is not strongly mixing.

For $x \in O_{1,1}^{(p, n+1)}=\left\{x \in D_{p, 1} \mid C_{p, 1}-x \subseteq C_{n, 1}\right\}$, and $v \in \mathcal{F}_{p, 1}$, we have by (Z.1) and by $i$ ) in Theorem 13

$$
C_{p+1,1}-(v+x) \subset C_{p+1}-x \subset C_{p, 1}-x \subset C_{n, 1} .
$$

Thus for any $x \in O_{1,1}^{(p, n+1)}$ and $v \in \mathcal{F}_{p, 1}$ we get $C_{p+1,1}-x \subset \tilde{C}(n, v)$. Then

$$
\nu(\tilde{C}(n, v)) \geq \sharp O_{1,1}^{(p, n+1)} \nu\left(C_{p+1,1}\right)=m_{1,1}^{(p, n+1)} \nu\left(C_{p+1,1}\right) .
$$

By Lemma 19, we obtain

$\lim \inf _{p \rightarrow \infty} \inf _{v \in \mathcal{F}_{p, 1}} \nu(\tilde{C}(n, v))$

$\geq \lim \inf _{p \rightarrow \infty} \frac{m_{1,1}^{(p, n+1)}}{\operatorname{vol}\left(D_{1, p}\right)} \nu\left(C_{p+1,1}\right) \operatorname{vol}\left(D_{1, p}\right)$

$\geq \nu\left(C_{n, 1}\right) c\left(\frac{K_{1}}{K_{2}}\right)^{d} \lim \inf _{p \rightarrow \infty} \nu\left(C_{p+1,1}\right) \operatorname{vol}\left(D_{1, p}\right)$

$\geq \nu\left(C_{n, 1}\right) c\left(\frac{K_{1}}{K_{2}}\right)^{d} \lim \inf _{p \rightarrow \infty} \nu\left(C_{p+1,1}\right) \operatorname{vol}\left(D_{1, p+1}\right) c$ by inequality 4.5

$\geq \nu\left(C_{n, 1}\right)\left(\frac{K_{1}}{K_{2}}\right)^{d} c^{2}>\nu\left(C_{n, 1}\right)^{2}$. 


\subsection{Subadditive ergodic theorem}

In section 4.2 we recall that the linearly repetitive systems are uniquely ergodic. Actually such systems satisfy also a subadditive ergodic theorem. Let $\mathcal{B}\left(\mathbb{R}^{d}\right)$ denotes the family of bounded subsets in $\mathbb{R}^{d}$. A real valued function $F: \mathcal{B}\left(\mathbb{R}^{d}\right) \rightarrow \mathcal{R}$ is called subadditive if

$$
F\left(Q_{1} \cup Q_{2}\right) \leq F\left(Q_{1}\right)+F\left(Q_{2}\right)
$$

for any disjoint sets $Q_{1}, Q_{2} \in \mathcal{B}\left(\mathbb{R}^{d}\right)$. For a Delone set $X$, the function $F$ is called $X$-invariant if

$$
F(Q)=F(Q+t) \quad \text { whenever } Q \in \mathcal{B}\left(\mathbb{R}^{d}\right) \text { and } t+(Q \cap X)=(t+Q) \cap X \text {. }
$$

For instance, given a patch $\mathrm{P}$ of the Delone set $X$, the function $B \in \mathcal{B}\left(\mathbb{R}^{d}\right) \mapsto$ $-X_{\mathrm{P}} \cap B$ where $X_{\mathrm{P}}$ denotes the set of occurences of the patches $\mathrm{P}$ in $X$, is a subadditive $X$-invariant function.

Theorem 20 ( $\mathrm{DL}, \mathrm{BBL})$. Let $X$ be a linearly repetitive Delone set in $\mathbb{R}^{d}$. Then $X$ satisfies the uniform ergodic theorem: i.e. for any $X$-invariant subadditive function $F$ and any nested sequence $\left(D_{n}\right)_{n}$ of $d$-cubes with side-lengths going to infinity as $n$ goes to infinity, the following limit exists

$$
\lim _{n \rightarrow+\infty} \frac{F\left(D_{n}\right)}{\operatorname{vol}\left(D_{n}\right)}
$$

and is independent of the sequence $\left(D_{n}\right)_{n}$.

It is then easy to deduce from this result that the associated dynamical system is uniquely ergodic. The converse is false, in DL, the authors give an example of a Sturmian sequence that does not satisfy the subadditive ergodic theorem. They prove also a more stronger form of this theorem.

The lower density $\underline{\nu}(\mathrm{P})$ of a $R$-patch $\mathrm{P}$ is the quantity

$$
\underline{\nu}(\mathrm{P}):=\liminf _{n \rightarrow \infty} \frac{\sharp X_{\mathrm{P}} \cap B_{n}(0)}{\operatorname{vol}\left(B_{n}(0)\right)} \operatorname{vol}\left(B_{R}(0)\right) .
$$

The results in $\mathrm{BBL}$, have this direct corollary.

Proposition 21. If $X$ is a repetitive $\left(r_{X}, R_{X}\right)$ Delone set verifying the uniform subadditive ergodic theorem, then $X$ satisfies positivity of weights: i.e.

$$
P \text { is a } \inf _{\text {patch }, R \geq R_{X}} \underline{\nu}(P)>0 \text {. }
$$

Notice that in dimension 1 , the positivity of weights property is sufficient to ensure the unique ergodictiy (see $[\mathrm{Bo}]$ ). Actually, one can deduce from Lemma 18 that a linearly repetitive Delone set satisfies the positivity of weights. 


\subsection{A characterization of linear repetitivity}

In Le02, D. Lenz characterizes the subshifts that admit a uniform subadditive ergodic Theorem by uniform positivity of weights. This can be considered as an averaged version of linear repetitivity. For Delone systems, it is shown in $\mathrm{Bes}, \mathrm{BBL}$ that the linear repetitivity is equivalent to positivity of weights plus some balancedness of the shape of patterns. For a Voronoï cell $V$ of a Delone set, let us define:

$$
\begin{aligned}
r_{i n t} & :=\sup \{r>0 ; V \text { contains a ball of radius } r\} . \\
R_{\text {ext }} & :=\inf \{R>0 ; V \text { is contained in a ball of radius } R\} .
\end{aligned}
$$

The distorsion of $V$ is the constant $\lambda(V):=R_{\text {ext }}(V) / r_{\text {int }}(V)$.

Theorem $22(\overline{\mathrm{BBL}})$. Let $X$ be an aperiodic Delone set in $\mathbb{R}^{d}$ of finite type. Then $X$ is linearly repetitive if and only if for any $R$-patch $P$ of $X, R>0$ : the set $X_{P}$ of occurences of $P$ is a $\left(r_{P}, R_{P}\right)$-Delone set such that

(i) $\sup _{P, x \in X_{P}} \lambda\left(V_{x}\right)<+\infty$ where $V_{x}$ denotes the Voronoï cell of $x$.

(ii) The Delone set $X$ satisfies the positivity of weights (see Proposition 21).

One can find in $\mathrm{BBL}$ another similar equivalent condition to linear repetitivity. Notice that in dimension $d=1$, the distorsion of any compact Voronoï cell is equal to 1 . Thus the condition (ii) is equivalent to the linear repetitivity.

For an aperiodic linearly repetitive Delone set, the properties (i)-(ii) arise from the properties recalled in the subsections 2.1 and 4.4

Let us also mention in Chapter On the non commutative geometry for tilings, a characterization of Sturmian sequences that are linearly repetitive by using metrics arising from the Connes distance.

\section{Factors of linearly repetitive system}

A factor map between two Delone systems $\left(\Omega_{1}, \mathbb{R}^{d}\right)$ and $\left(\Omega_{2}, \mathbb{R}^{d}\right)$ is a continuous surjective map $\pi: \Omega_{1} \rightarrow \Omega_{2}$ such that $\pi(X-v)=\pi(X)-v$, for every $X \in \Omega_{1}$ and $v \in \mathbb{R}^{d}$.

In symbolic dynamics it is well-known that topological factor maps between subshifts are always given by sliding-block-codes. An equivalent notion for the Delone system is the local derivability: i.e. there exists a constant $s_{0}>0$ such that for any radius $R>0$, if two Delone sets $X, Y \in \Omega_{1}$ satisfy $X \cap B_{R+s_{0}}(0)=Y \cap B_{R+s_{0}}(0)$ then $\pi(X) \cap B_{R}(0)=\pi(Y) \cap B_{R}(0)$. However there are examples of factor maps on Delone systems that are not slidingblock codes $([\underline{\mathrm{Pe}}, \underline{\mathrm{RS}}])$. Nevertheless, the following lemma shows that factor maps between Delone systems are not far from being sliding-block-codes. Similar results can be found in [CD, CDP, $\mathrm{HRS}$.

Lemma 23. Let $X_{1}$ and $X_{2}$ be two Delone sets. Suppose $X_{1}$ has finite local complexity and $\pi: \Omega_{X_{1}} \rightarrow \Omega_{X_{2}}$ is a factor map. Then, there exists a constant 
$s_{0}>0$ such that for every $\varepsilon>0$, there exists $R_{\varepsilon}>0$ satisfying the following: For any $R \geq R_{\varepsilon}$, if $X$ and $X^{\prime}$ in $\Omega_{X_{1}}$ satisfy

$$
X \cap B_{R+s_{0}}(0)=X^{\prime} \cap B_{R+s_{0}}(0),
$$

then

$$
(\pi(X)-v) \cap B_{R}(0)=\pi\left(X^{\prime}\right) \cap B_{R}(0)
$$

for some $v \in B_{\varepsilon}(0)$.

Proof. The Delone set $X_{2}$ has also finite local complexity because $\Omega_{X_{2}}$ is compact. Let $r_{0}$ and $R_{0}$ be positive constants such that $X_{2}$ is a $\left(r_{0}, R_{0}\right)$ Delone set. Since all the elements of $\Omega_{X_{2}}$ are $\left(r_{0}, R_{0}\right)$-Delone sets, if two different points $y_{1}, y_{2}$ of $\mathbb{R}^{d}$ satisfy $\left(X-y_{1}\right) \cap B_{R}(a)=\left(X-y_{2}\right) \cap B_{R}(a)$ for some $X \in \Omega_{X_{2}}, a \in \mathbb{R}^{d}$ and $R>R_{0}$, then $\left\|y_{1}-y_{2}\right\| \geq \frac{r_{0}}{2}$ (for the details see So1]).

Let $0<\delta_{0}<\min \left\{\frac{r_{0}}{4}, \frac{1}{R_{0}}\right\}$. Since $\pi$ is uniformly continuous, there exists $s_{0}>1$ such that if $X$ and $X^{\prime}$ in $\Omega_{X_{1}}$ verify $X \cap B_{s_{0}}(0)=X^{\prime} \cap B_{s_{0}}(0)$ then

$$
(\pi(X)-v) \cap B_{\frac{1}{\delta_{0}}}(0)=\pi\left(X^{\prime}\right) \cap B_{\frac{1}{\delta_{0}}}(0),
$$

for some $v \in B_{\delta_{0}}(0)$. Let $0<\varepsilon<\delta_{0}$. By uniform continuity of $\pi$, there exists $0<\delta<\frac{1}{s_{0}}$ such that if $X$ and $X^{\prime}$ in $\Omega_{X_{1}}$ verify $X \cap B_{\frac{1}{\delta}}(0)=X^{\prime} \cap B_{\frac{1}{\delta}}(0)$ then

$$
(\pi(X)-v) \cap B_{\frac{1}{\varepsilon}}(0)=\pi\left(X^{\prime}\right) \cap B_{\frac{1}{\varepsilon}}(0),
$$

for some $v \in B_{\varepsilon}(0)$. Now fix $R \geq R_{\varepsilon}=\frac{1}{\delta}-s_{0}$, and let $X$ and $X^{\prime}$ be two Delone sets in $\Omega_{X_{1}}$ satisfying

$$
X \cap B_{R+s_{0}}(0)=X^{\prime} \cap B_{R+s_{0}}(0) .
$$

Observe that $X$ and $X^{\prime}$ satisfy (5.1), and $(X-a) \cap B_{s_{0}}(0)=\left(X^{\prime}-a\right) \cap B_{s_{0}}(0)$, for every $a$ in $B_{R}(0)$. The choice of $s_{0}$ ensures that

$$
(\pi(X)-a-t(a)) \cap B_{\frac{1}{\delta_{0}}}(0)=\left(\pi\left(X^{\prime}\right)-a\right) \cap B_{\frac{1}{\delta_{0}}}(0),
$$

for some $t(a) \in B_{\delta_{0}}(0)$. Let us prove the map $a \mapsto t(a)$ is locally constant. For $a \in B_{R}(0)$, let $0<s_{a}<\frac{1}{\delta_{0}}-R_{0}$ be such that $B_{s_{a}}(a) \subseteq B_{R}(0)$. Every $a^{\prime} \in B_{s_{a}}(0)$ verifies $B_{\frac{1}{\delta_{0}}-\left\|a^{\prime}\right\|}\left(-a^{\prime}\right) \subset B_{\frac{1}{\delta_{0}}}(0)$. Let $a^{\prime} \in B_{s_{a}}(0)$. This inclusion and (5.3) imply

$$
\left(\pi(X)-a-a^{\prime}-t(a)\right) \cap B_{\frac{1}{\delta_{0}}-\left\|a^{\prime}\right\|}\left(-a^{\prime}\right)=\left(\pi\left(X^{\prime}\right)-a-a^{\prime}\right) \cap B_{\frac{1}{\delta_{0}}}-\left\|a^{\prime}\right\|\left(-a^{\prime}\right) .
$$

On the other hand, from the definition of the map $a \mapsto t(a)$ we deduce

$$
\left(\pi(X)-a-a^{\prime}-t\left(a+a^{\prime}\right)\right) \cap B_{\frac{1}{\delta_{0}}}(0)=\left(\pi\left(X^{\prime}\right)-a-a^{\prime}\right) \cap B_{\frac{1}{\delta_{0}}}(0),
$$

which implies

$$
\left(\pi(X)-a-a^{\prime}-t\left(a+a^{\prime}\right)\right) \cap B_{\frac{1}{\delta_{0}}-\left\|a^{\prime}\right\|}\left(-a^{\prime}\right)=\left(\pi\left(X^{\prime}\right)-a-a^{\prime}\right) \cap B_{\frac{1}{\delta_{0}}}-\left\|a^{\prime}\right\|\left(-a^{\prime}\right) .
$$

Since $\left\|t(a)-t\left(a+a^{\prime}\right)\right\| \leq \frac{r_{0}}{2}$, from equations (5.4), (5.5) and the remark of the beginning of the proof we conclude $t(a)=t\left(a+a^{\prime}\right)$ for every $a^{\prime} \in B_{s}(0)$. Therefore the map $a \mapsto t(a)$ is constant on $B_{s_{a}}(a)$. 
Furthermore, due to $\delta_{0}>\varepsilon$ and $(5.2)$, Equation (5.1) implies there exists $v \in B_{\varepsilon}(0)$ such that

$$
(\pi(X)-v) \cap B_{\frac{1}{\delta_{0}}}(0)=\pi\left(X^{\prime}\right) \cap B_{\frac{1}{\delta_{0}}}(0) .
$$

For $a=0$, from 5.3 and 5.6 we have that $t(0)=v$ or $\|v-t(0)\| \geq \frac{r_{0}}{2}$. Since $\|t(0)-v\| \leq \delta_{0}+\varepsilon<2 \delta_{0}<\frac{T_{0}}{2}$, we conclude $t(0)=v$ and then $t(a)=v$ for every $a \in B_{R}(0)$. This property together with (5.3) and (5.6) imply that

$$
(\pi(X)-v) \cap B_{R}(0)=\pi\left(X^{\prime}\right) \cap B_{R}(0) .
$$

This conclude the proof.

Lemma 24 (CD Lem. 3). Let $X_{1}$ and $X_{2}$ be two Delone sets with finite local complexity. If $\pi: \Omega_{X_{1}} \rightarrow \Omega_{X_{2}}$ is a factor map and $X_{1}$ is linearly repetitive, then $\left(\Omega_{X_{2}}, \mathbb{R}^{d}\right)$ is linearly repetitive.

Proof. Let $X \in \Omega_{X_{1}}$. Consider $0<\varepsilon<1$ and $s_{0}, R(\varepsilon)>0$ the positive constants of Lemma 23 associated to $\varepsilon$. Since $X$ is linearly repetitive with some constant $L$, for any $y \in \mathbb{R}^{d}$ there exists $v \in B_{L\left(R+s_{0}\right)}(y)$ such that $(X-v) \cap B_{R+s_{0}}(0)=X \cap B_{R+s_{0}}(0)$. From Lemma 23 there exists $t \in B_{\varepsilon}(0)$ such that $(\pi(X)-v-t) \cap B_{R}(0)=\pi(X) \cap B_{R}(0)$. This implies that any ball of radius $L\left(R+s_{0}\right)+2 \varepsilon$ in $\pi(X)$ contains a copy of $\pi(X) \cap B_{R}(0)$. Since $L s_{0}+2 \varepsilon$ is smaller than the constant $L s_{0}+2$, it follows that $\pi(X)$ is linearly repetitive.

Actually from the proofs of Lemmas 4 and 24 we can get an uniform bound on the linear repetitivity constant of the factor system.

Lemma 25. Let $X_{1}$ and $X_{2}$ be two Delone sets with finite local complexity. If $\pi: \Omega_{X_{1}} \rightarrow \Omega_{X_{2}}$ is a factor map and $X_{1}$ is linearly repetitive with constant $L>1$, then there exists $R_{\pi}>0$ such that for every $R>R_{\pi}$ and every $R$ patch $P$ of $X_{2}$, a copy of $P$ appears in every ball of radius $3 L R$ of $X_{2}$ and any two occurrences of $P$ in $X_{2}$ are at distance at least $R / 4 L$.

\subsection{Finite number of aperiodic Delone systems as factors}

The aim of this section is to prove the following theorem that is a generalization of a result in [Du1] in the context of subshifts.

Theorem 26 (CDP Theo. 12). Let $L>1, d \geq 1$. There exists a constant $N(L, d)$ such that any linearly repetitive Delone set $X$ of $\mathbb{R}^{d}$ with constant $L$, has at most $N(L, d)$ aperiodic Delone system factors of $\left(\Omega_{X}, \mathbb{R}^{d}\right)$ up to conjugacy.

The bound $N(L, d)$ is essentially due to the constants arising in the lemmas 6 and 7. The proof relies on a generalization of these lemmas and on the specific structure of the factor maps for linearly repetitive Delone systems.

The next result says that factor maps between linearly repetitive Delone systems are finite-to-one. A proof of that result in the context of subshifts 
and Delone systems can be found in [Du1 and in [CDP, Proposition 5] respectively. Here we include the proof in the case where the factor map is a sliding-block-code.

Proposition 27. Let $X$ be a linearly repetitive Delone set with constant L. There exists a constant $C>0$ (depending only on $L$ ) such that If $X^{\prime}$ is an aperiodic Delone set and $\pi:\left(\Omega_{X}, \mathbb{R}^{d}\right) \rightarrow\left(\Omega_{X^{\prime}}, \mathbb{R}^{d}\right)$ is a factor map, then for every $Y \in \Omega_{X^{\prime}}$, the fiber $\pi^{-1}(\{Y\})$ contains at most $C$ elements.

Proof. For simplicity we will assume that $\pi$ is a sliding-block-code. That means there exists $s_{0}>0$ such that if $X_{1}$ and $X_{2} \in \Omega_{X}$ verify $X_{1} \cap$ $B_{R+s_{0}}(0)=X_{2} \cap B_{R+s_{0}}(0)$ for an $R>0$, then $\pi\left(X_{1}\right) \cap B_{R}(0)=\pi\left(X_{2}\right) \cap B_{R}(0)$. From Lemma 24 the Delone set $X^{\prime}$ is linearly repetitive, and if $R$ is sufficiently large, Lemma 25 implies that for any $x \in \mathbb{R}^{d}$ a copy of the patch $X^{\prime} \cap B_{R}(x)$ appears in $X^{\prime} \cap B_{3 L R}(y)$, for every $y \in \mathbb{R}^{d}$. Let $Y \in \Omega_{X^{\prime}}$ and $X_{1}, \cdots, X_{n}$ be different Delone sets in $\in \pi^{-1}(\{Y\})$. Because these Delone sets are different, for every sufficiently large $R$, the patches $X_{i} \cap B_{R}(0)$ are pairwise distinct. Linearly repetitivity of $X$ ensures the existence of points $v_{1}, \cdots, v_{n} \in B_{L R}(0)$ such that each $X-v_{i} \cap B_{R}(0)$ is a copy of $X_{i} \cap B_{R}(0)$, for every $1 \leq i \leq n$. This implies that $\pi(X)-v_{i} \cap B_{R-s_{0}}(0)=Y \cap B_{R-s_{0}}(0)$. From this and Lemma 25 we get that $\left\|v_{i}-v_{j}\right\| \geq \frac{R-s_{0}}{4 L}$, from which we deduce that $n \leq C$, where $C$ is a constant that depends only on $L$.

The following proposition is a straightforward generalization of Lemma 21 in [Du1. A proof in our setting can be found in [CDP, Proposition 6]. Here we omit the proof.

Proposition 28. Let $\left(\Omega, \mathbb{R}^{d}\right)$ be a minimal Delone system and $\phi_{1}:\left(\Omega, \mathbb{R}^{d}\right) \rightarrow$ $\left(\Omega_{1}, \mathbb{R}^{d}\right), \phi_{2}:\left(\Omega, \mathbb{R}^{d}\right) \rightarrow\left(\Omega_{2}, \mathbb{R}^{d}\right)$ be two factor maps. Suppose that $\left(\Omega_{2}, \mathbb{R}^{d}\right)$ is non periodic and $\phi_{1}$ is finite-to-one. If there exist $X, Y \in \Omega$ and $v \in \mathbb{R}^{d}$ such that $\phi_{1}(X)=\phi_{1}(Y)$ and $\phi_{2}(X)=\phi_{2}(Y-v)$, then $v=0$.

We have already defined the notion of return vector of a patch, now let us define the notion of return vector of a Voronoï cell of a patch. For a patch $\mathrm{P}$ of $X$ and $v \in X_{\mathrm{P}}, V_{\mathrm{P}, v}$ denotes the Vornoï cell of the point $v$ of the Delone set $X_{\mathrm{P}}$. We say that $w \in \mathbb{R}^{d}$ is a return vector of $V_{\mathrm{P}, v} \cap X$ if $(X-w) \cap V_{\mathrm{P}, v}=X \cap V_{\mathrm{P}, v}$. We set for $n \geq 1, v \in X_{\mathrm{P}}$,

$$
\mathrm{P}_{n, w, v} \text { the patch }(X-w-v) \cap B_{L^{n} R}(0) \text {. }
$$

Notice that $\mathrm{P}_{n, w, v}+v+w$ is a patch of $X$. When there is no confusion about $n$ and $v$, we write $\mathrm{P}_{w}$ instead of $\mathrm{P}_{n, w, v}$.

The following lemma generalizes Lemma 6

Lemma 29. Let $n \in \mathbb{N}^{*}$ and $X$ be an aperiodic linearly repetitive Delone set with constant $L$. There exists a constant $C(n, L)>0$ such that for every sufficiently large $R>0$ and every $R$-patch $P$, the collection $\left\{P_{n, w, v}\right.$ : $w$ is a return vector of $\left.V_{P, v} \cap X\right\}$ has at most $C(n, L)$ elements, for every $v \in X_{P}$. 
Proof. Let $\mathrm{P}=X \cap B_{R}\left(x_{\mathrm{P}}\right)$ and $v \in X_{\mathrm{P}}$. Lemma 4 implies that the Voronoï cell $V_{\mathrm{P}, v}$ contains the ball $B_{\frac{R}{2(L+1)}}(v)$. Then for every pair of return vectors $u$ and $w$ of $V_{\mathrm{P}, v}$, the patches $\mathrm{P}_{u}$ and $\mathrm{P}_{w}$ coincides at the ball $B_{\frac{R}{2(L+1)}}(0)$. The proof concludes using the fact that in $X \cap B_{2 L\left(L^{n} R\right)}(0)$ there is at least one copy of each patch $\mathrm{P}_{w}, \mathrm{P}_{u}$ and appliying Lemma 4 to the return vectors of the patch $\mathrm{P}_{w} \cap B_{\frac{R}{2(L+1)}}(0)$.

Proof of Theorem 26. It is enough to suppose that $X$ is an aperiodic linearly repetitive Delone set with constant $L>1$. Let $n \in \mathbb{N}$ be such that

$$
L^{n}-1-12 L-64 L^{2}>1 .
$$

We call $M(n, L)$ the number of coverings of a set with $c(L) c(n, L)$ elements, where $c(L)$ and $c(n, L)$ are the constants of Lemma 6 and Lemma 29 respectively. For every $1 \leq i \leq M(n, L)+1$, let $X_{i}$ be a non periodic Delone set such that there exists a topological factor map $\pi_{i}: \Omega_{X} \rightarrow \Omega_{X_{i}}$, and let $X_{0}=X$. We will show there exist $1 \leq i<j \leq M(n, L)+1$ such that $\left(\Omega_{X_{i}}, \mathbb{R}^{d}\right)$ and $\left(\Omega_{X_{j}}, \mathbb{R}^{d}\right)$ are conjugate.

Since $M(n, L)$ is finite, we can take the same constant $s_{0}>0$ and $R_{\pi}$ of Lemmas 23 and 25 respectively, associated to each $\pi_{i}$. Fix $0<\varepsilon<1$. Let $R>\sup \left\{s_{0}, R_{\pi}+\varepsilon, 17 L\right\}$ be sufficiently large such that Lemma 6 and Lemma 29 are applicable to $R$-patches of $X$, and such that Lemma 23 is applicable to $\varepsilon$ and each $\pi_{i}$.

Consider the patch $\mathrm{P}=B_{R}(0) \cap X$, and $v_{1}, \cdots, v_{N} \in X_{\mathrm{P}}$ such that for every $v \in X_{\mathrm{P}}$, there exist $1 \leq i \leq N$ and $u \in \mathbb{R}^{d}$ satisfying $V_{\mathrm{P}, v} \cap X=$ $\left(V_{\mathrm{P}, v_{i}} \cap X\right)+u$. Roughly speaking, every set of the kind $V_{\mathrm{P}, v} \cap X$ is a translated of some set $V_{\mathrm{P}, v_{i}} \cap X$. Since $R>R_{1}$, Lemma 6 ensures $N \leq c(L)$.

For every $1 \leq j \leq N$, let $w_{j, 1}, \cdots, w_{j, m_{j}}$ be return vectors of $V_{\mathrm{P}, v_{j}} \cap X$, chosen in order that for every return vector $w$ of $V_{\mathrm{P}, v_{j}} \cap X$, there exists $1 \leq i \leq m_{j}$ such that $\mathrm{P}_{n, w, v_{j}}$ is equal to $\mathrm{P}_{n, w_{j, i}, v_{j}}=: \mathrm{P}_{w_{j, i}}$. Since $R>R_{1}$, Lemma 29 implies that $m_{j} \leq c(n, L)$, for every $1 \leq j \leq N$. Therefore, the collection

$$
\mathcal{F}=\left\{\mathrm{P}_{w_{j, l}}: 1 \leq l \leq m_{j}, 1 \leq j \leq N\right\}
$$

contains at most $c(L) c(n, L)$ elements.

We define $R^{\prime}=\left(L^{n}-1\right) R-\varepsilon-4 L R$. The choice of $n$ ensures that $R^{\prime}>0$.

For every $1 \leq i \leq M(n, L)+1$, we define the following relation on $\mathcal{F}$ :

$\mathrm{P}_{w_{j, l}} \leftrightarrow_{i} \mathrm{P}_{w_{k, m}}$ if and only if for every $X^{\prime}, X^{\prime \prime} \in \Omega_{X}$ such that $X^{\prime} \cap$ $B_{L^{n} R}(0)=\mathrm{P}_{w_{j, l}}$ and $X^{\prime \prime} \cap B_{L^{n} R}(0)=\mathrm{P}_{w_{k, m}}$, there exist $v \in B_{2 \varepsilon}(0)$ and $w \in B_{4 L R}(0)$ such that $\pi_{i}\left(X^{\prime}\right) \cap B_{R^{\prime}}(0)=\left(\pi_{i}\left(X^{\prime \prime}\right)+v+w\right) \cap B_{R^{\prime}}(0)$.

Since $L^{n} R-s_{0} \geq\left(L^{n}-1\right) R \geq R$, from Lemma 23 it follows this relation is reflexive, so non empty. Since the cardinal of $\mathcal{F}$ is bounded by $c(L) c(n, L)$, there are at most $M(n, L)$ different relations of this kind. So, there exist $1 \leq i<j<M(n, L)+1$ such that $\leftrightarrow_{i}=\leftrightarrow_{j}$. 
In the sequel, we will prove that $\left(\Omega_{X_{i}}, \mathbb{R}^{d}\right)$ and $\left(\Omega_{X_{j}}, \mathbb{R}^{d}\right)$ are conjugate. For that, it is sufficient to show that if $Y, Z \in \Omega_{X}$ are such that $\pi_{i}(Y)=\pi_{i}(Z)$ then $\pi_{j}(Y)=\pi_{j}(Z)$.

Let $Y$ and $Z$ be two Delone sets in $\Omega_{X}$ such that $\pi_{i}(Y)=\pi_{i}(Z)$. Without loss of generality, we can suppose that 0 is an occurrence of $\mathrm{P}$ in $Y$ and in $Z-u_{0}$, where $u_{0}$ is some point in $B_{4 L R}(0)$. The patches of $Y$ and $Z$ are translated of the patches of $X$. This implies there exist $1 \leq q_{0}, r_{0} \leq N$ such that

$$
Y \cap B_{L^{n} R}(0)=\mathrm{P}_{w_{q_{0}, l_{0}}} \text { and }\left(Z-u_{0}\right) \cap B_{L^{n} R}(0)=\mathrm{P}_{w_{r_{0}, k_{0}}},
$$

for some $1 \leq l_{0} \leq m_{q_{0}}$ and $1 \leq k_{0} \leq m_{r_{0}}$.

It is possible to show that $\mathrm{P}_{w_{q_{0}, l_{0}}} \leftrightarrow_{i} \mathrm{P}_{w_{r_{0}, k_{0}}}$ and $\mathrm{P}_{w_{q_{0}, l_{0}}} \leftrightarrow_{j} \mathrm{P}_{w_{r_{0}, k_{0}}}$ for $R$ sufficiently large (see Claim 1 in the proof of [CDP, Theorem 12]).

Let $s$ be any other occurrence of $\mathrm{P}$ in $Y$. Repeating the same argument for $Y+s$ and $Z+s$, we deduce there exist $u_{s} \in B_{4 L R}(0)$ and $1 \leq q_{s}, r_{s} \leq N$ such that

$$
(Y+s) \cap B_{L^{n} R}(0)=\mathrm{P}_{w_{q_{s}, l_{s}}} \text { and }\left(Z+s-u_{s}\right) \cap B_{L^{n} R}(0)=\mathrm{P}_{w_{r_{s}, k_{s}}},
$$

for some $1 \leq l_{s} \leq m_{q_{s}}$ and $1 \leq k_{s} \leq m_{r_{s}}$. Then we get $\mathrm{P}_{w_{q_{s}, l_{s}}} \leftrightarrow_{j} \mathrm{P}_{w_{r_{s}, k_{s}}}$. This implies there exist $t_{s} \in B_{2 \varepsilon}(0)$ and $w_{s} \in B_{4 L R}(0)$ such that

$$
\pi_{j}(Y+s) \cap B_{R^{\prime}}(0)=\left(\pi_{j}\left(Z+s-u_{s}\right)+t_{s}+w_{s}\right) \cap B_{R^{\prime}}(0) .
$$

Showing that $w_{s}-u_{s}+t_{s}$ does not depend on $s$ (see Claim 2 in the proof of CDP , Theorem 12]), we get there exists $y \in \mathbb{R}^{d}$ such that for every occurrence $s$ of $\mathrm{P}$ in $Y$,

$$
\begin{aligned}
\pi_{j}(Y+s) \cap B_{R^{\prime}}(0) & =\left(\pi_{j}(Z+s)+y\right) \cap B_{R^{\prime}}(0), \text { and then } \\
\pi_{j}(Y) \cap B_{R^{\prime}}(s) & =\left(\pi_{j}(Z)+y\right) \cap B_{R^{\prime}}(s) .
\end{aligned}
$$

The diameter of the Voronoï cells of $\mathrm{P}$ is less than $4 L R$ (see 2.1), which is less than $R^{\prime}$. Hence,

$$
\pi_{j}(Y)=\pi_{j}(Z)+y .
$$

We conclude with Proposition 27 and 28 .

\subsection{Factors on groups and cocycles}

Cocycles and cohomological equations play an important role in the study of factors dynamical systems, time change for flows orbit equivalence, ... We adapt this notion to the context of Delone system $\left(\Omega, \mathbb{R}^{d}\right)$. Let $G$ denotes the group $\mathbb{R}^{m}$ or $\mathbb{T}^{m}=\mathbb{R}^{m} / \mathbb{Z}^{m}$. A continuous $G$-cocycle is a continuous function $\alpha: \Omega \times \mathbb{R}^{d} \rightarrow G$ so that

$$
\alpha(Y, v+w)=\alpha(Y, v)+\alpha(Y+v, w) \quad \text { for all } Y \in \Omega, v, w \in \mathbb{R}^{d} .
$$

An important question which appears in many problems, is to known if the cohomological equation

$$
\alpha(Y, v)=\psi(Y+x)-\psi(Y)
$$

has a measurable, continuous solution $\psi: \Omega \rightarrow G$. This solution is called a transfer function and if it exists, $\alpha$ is called a coboundary. 
In section 5.2 .2 we will give a necessary and sufficient condition to find solutions to the cohomological equation for linearly repetitive Delone systems. We will focus on transversally locally constant cocycle $\alpha$, i.e.: if there exists $r, R>0$ such that for any $Y, Y^{\prime} \in \Omega$ and $x \in B_{R}(0)$,

$$
\text { if } Y \cap B_{R}(0)=Y^{\prime} \cap B_{R}(0) \text { then } \alpha(Y, x)=\alpha\left(Y^{\prime}, x\right) \text {. }
$$

More generally a cocycle $\alpha$ is transversally Hölder if there exist constants $K>0$ and $\delta \in(0,1)$ such that for all $r>0, Y, Y^{\prime} \in \Omega$ and $x \in B_{r}(0)$,

$$
\text { if } Y \cap B_{R}(0)=Y^{\prime} \cap B_{R}(0) \text { then }\left|\alpha(Y, x)-\alpha\left(Y^{\prime}, x\right)\right| \leq K r^{-\delta} \text {. }
$$

5.2.1. Examples of cohomological equations. Let us see first some dynamical problems where the cohomological equation appears.

Let us denote by $\langle.,$.$\rangle the usual inner product in \mathbb{R}^{d}$ and $\mu$ be an ergodic $\mathbb{R}^{d}$ invariant probability measure on the hull $\Omega$. A vector $\lambda \in \mathbb{R}^{d}$ is a measurable eigenvalue of the system $\left(\Omega, \mathbb{R}^{d}\right)$ if there exists a measurable function $\psi: \Omega \rightarrow \mathbb{S}^{1}$ such that

$$
\psi(Y+v)=e^{2 i \pi\langle\lambda, v\rangle} \psi(Y) \text { for all } v \in \mathbb{R}^{d} \text { and } \mu-\text { a.e. } Y \in \Omega .
$$

If the function $\psi$ is continuous, then $\lambda$ is called a continuous eigenvalue. The map $(Y, v) \mapsto e^{2 i \pi\langle\lambda, v\rangle}$ is a $\mathbb{S}^{1}$-cocycle over $\left(\Omega, \mathbb{R}^{d}\right)$. Then passing in additive notation $\mathbb{T}^{1}$, we have $\lambda$ is a measurable (resp. continuous) eigenvalue of $\left(\Omega, \mathbb{R}^{d}\right)$ if and only if there is a measurable (resp. continuous) solution $\psi: \Omega \rightarrow \mathbb{T}^{1}$ to the cohomological equation

$$
\langle\lambda, v\rangle=\psi(Y+v)-\psi(Y) \bmod \mathbb{Z} .
$$

A continuous eigenvalue gives then a topological factor on the closure of an orbit in the 1 dimensional torus $\mathbb{T}^{1}$. More generally, one can consider the closure $\mathbf{O}$ of an orbit of a $n$-rotations on the $n$-torus $\mathbb{T}^{n}, n \leq d$, that are factors of the system $\left(\Omega, \mathbb{R}^{d}\right)$. More precisely, take $\theta=\left(\theta_{1}, \ldots, \theta_{n}\right) \in \mathbb{R}^{n}$ and let $\mathcal{A}: \mathbb{R}^{d} \times \mathbb{T}^{n} \rightarrow \mathbb{T}^{n}$ be the continuous action defined by

$$
\mathcal{A}(v, x)=x+[v, \theta] \quad \text { where }[v, \theta]=\left(v_{1} \theta_{1}, \ldots, v_{n} \theta_{n}\right) .
$$

The map $(Y, v) \mapsto[v, \theta]$ is a $\mathbb{T}^{n}$-cocycle over $\left(\Omega, \mathbb{R}^{d}\right)$. It is standard to show that the system $(\mathbf{O}, \mathcal{A})$ is a topological factor of $\left(\Omega, \mathbb{R}^{d}\right)$ if and only there exists a continuous solution $\psi: \Omega \rightarrow \mathbb{T}^{n}$ to the cohomological equation

$$
[v, \theta]=\psi(Y+v)-\psi(Y) .
$$

5.2.2. Characterization of continuous coboundary. A seminal work for the characterization of continuous eigenvalues of symbolic systems given by a primitive substitution, is in [H]. The authors of [CDHM, BDM1] generalize these results to the linearly recurrent symbolic systems and to finite rank systems in BDM2]. An extension to $\mathbb{Z}^{d}$-action on a Cantor set is presented in CGM]. We present here a part of the results in [C] that treat only continuous cocycles and generalizes the results of [CGM]. 
For a box decomposition $\mathcal{B}=\left\{C_{i}\left[D_{i}\right]\right\}_{i=1}^{t}$ (see section 3.2.1), a first retrun vector to $C=\cup_{i} C_{i}$ is a vector $v \in \mathbb{R}^{d}$ with label $(i, j) \in\{1, \ldots, t\}^{2}$, such that

$$
C_{i}-v \cap C_{j} \neq \emptyset \text { and } C_{i}\left[D_{i}\right] \cap C_{j}\left[D_{j}\right] \neq \emptyset .
$$

We denote by $\mathcal{F}$ the set of first return vectors to $C$ associated with $\mathcal{B}$, and by $C(v)=C_{i} \cap\left(C_{j}+v\right)$ for a return vector $v$ with label $(i, j)$.

A tower system $\left(\mathcal{B}_{n}=\left\{C_{n, i}\left[D_{n, i}\right]\right\}_{i=1}^{t_{n}}\right)_{n}$ is well distributed if it satisfies the properties $i$ )-iii) in Theorem 13 and moreover for every $n \geq 0$, and every first return vector $v \in \mathcal{F}_{n}$ with label $(i, j)$ there are $x$ and $x^{\prime}$ in $D_{n+1,1}$ such that for $X \in \bigcap_{n} C_{n}, X-x \in C_{n, i}$ and $X-x^{\prime} \in C_{n, j}$ and $v=x-x^{\prime}$.

It is straightforward to check that this extra condition holds when each $D_{n+1, i}$ is big enough: more precisely when for any $n \geq 0$

$$
r_{\text {int }}\left(\mathcal{B}_{n+1}\right) \geq\left(R_{\text {rec }}\left(\mathcal{B}_{n}\right)+R_{\text {ext }}\left(\mathcal{B}_{n}\right)\right) L \geq M_{X}\left(R_{\text {rec }}\left(\mathcal{B}_{n}\right)+R_{\text {ext }}\left(\mathcal{B}_{n}\right)\right) .
$$

For a linearly repetitive Delone set $X$, it is direct to check that for a constant $K$ big enough, the tower system given by Theorem 13 , satisfies the inequality 5.8. Thus any linearly repetitive Delone system admits a well distributed tower system. In the following $|\cdot|$ denotes the usual distance to the origin when $G=\mathbb{R}^{m}$ or $\mathbb{T}^{m}$.

Theorem $30(\mathrm{C})$. Let $X$ be a linearly repetitive Delone set in $\mathbb{R}^{d}$, $G$ be the group $\mathbb{R}^{m}$ or $\mathbb{T}^{m}$, $\alpha$ be a continuous $G$-cocycle over $\left(\Omega, \mathbb{R}^{d}\right)$, and $\left(\mathcal{B}_{n}\right)_{n \geq 0}$ be a well-distributed tower system. Then $\alpha$ is a tansversally Hölder coboundary with continuous transfer function if and only if the series

$$
\sum_{n \geq 0} \sup _{\substack{v \in \mathcal{F}_{n} \\ \omega \in C_{n}(v)}}|\alpha(\omega, v)|
$$

converges, where each $\mathcal{F}_{n}$ denotes the set of first return vectors associated with $\mathcal{B}_{n}$.

In [C] appears also similar necessary conditions for a cocycle to be a coboundary on a general Delone system (without the assumption of linear repetitivity).

5.2.3. Characterization of the measurable eigenvalues. To be more complete on the problem of eigenvalues, let us mention that a characterization of measurable eigenvalues of linearly recurrent Cantor system is given in BDM1] and measurable coboundary for linearly repetitive Delone systems in [C0].

Theorem $31(\mathrm{C} 0)$. Let $\left(\Omega, \mathbb{R}^{d}\right)$ be a linearly repetitive Delone system, $\mu$ be the unique invariant measure, $G$ be the group $\mathbb{R}^{m}$ or $\mathbb{T}^{m}$, $\alpha$ be a transversally locally constant $G$-cocycle over $\left(\Omega, \mathbb{R}^{d}\right)$, and $\left(\mathcal{B}_{n}\right)_{n \geq 0}$ be a tower system well distributed. Then the following are equivalent.

1. The series $\sum_{n \geq 0} \sup _{\substack{v \in \mathcal{F}_{n} \\ \omega \in C_{n}(v)}}|\alpha(\omega, v)|^{2}$ converges, where each $\mathcal{F}_{n}$ denotes the set of first return vectors associated with $\mathcal{B}_{n}$. 
2. There exists a measurable function $\psi: \Omega \rightarrow G$ such that for $\mu$-a-e $X \in$ $\Omega$,

$$
\alpha(X, v)=\psi(X-v)-\psi(X), \quad \text { for all } v \in \mathbb{R}^{d} .
$$

Moreover $\psi \in L^{2}\left(\Omega, \mathbb{R}^{m}, \mu\right)$ when $G=\mathbb{R}^{m}$.

\section{Bi-Lipschitz equivalence to a lattice}

Let $X_{1}$ and $X_{2}$ be two Delone sets in $\mathbb{R}^{d}$. We say that they are bi-Lipschitz equivalent if there exists a homeomorphism $\phi: X_{1} \rightarrow X_{2}$ and a constant $\Delta \geq 1$ such that $\forall x, x^{\prime} \in X, x \neq x^{\prime}$

$$
\frac{1}{\Delta} \leq \frac{\left\|\phi(x)-\phi\left(x^{\prime}\right)\right\|}{\left\|x-x^{\prime}\right\|} \leq \Delta .
$$

The map $\phi$ is then called a bi-Lipschitz homeomorphism between $X_{1}$ and $X_{2}$.

The problem to know whether two Delone sets are bi-Lipschitz equivalent was raised by Gromov in Gro93, and boiled down in Toledo's review Tol] to the following question for the 2-dimensional Euclidean space: Is every Delone set in $\mathbb{R}^{2}$ bi-Lipschitz equivalent to $\mathbb{Z}^{2}$ ? Counterexamples to this question were given independently by Burago and Kleiner BK and McMullen [McM]. Moreover, McMullen also showed that when relaxing the bi-Lipschitz condition to a Hölder one, all Delone set (with or without finite local complexity) in $\mathbb{R}^{d}$ are equivalent. Later, Burago and Kleiner BK1 gave a sufficient condition for a Delone set to be bi-Lipschitz equivalent to $\mathbb{Z}^{2}$ and asked the following question: If one forms a Delone set in the plane by placing a point in the center of each tile of a Penrose tiling, is the resulting set bi-Lipschitz equivalent to $\mathbb{Z}^{2}$ ? They studied the more general question of knowing whether a Delone set arising from a cut-and-project tiling is bi-Lipschitz equivalent to $\mathbb{Z}^{2}$ (recall that the Penrose tiling is also a cut-and project tiling [Bru]) and solved it in some cases that do not include the case of Penrose tilings, thus leaving the former question open. Recently, Solomon [Solo] gave a positive answer for Penrose tiling by using the fact that it can be constructed using substitutions. In fact, Solomon proved that each Delone set arising from a primitive self-similar tiling in $\mathbb{R}^{2}$ is bi-Lipschitz to $\mathbb{Z}^{2}$.

The following result was proved in ACG1.

Theorem 32. Every linearly repetitive Delone set in $\mathbb{R}^{d}$ is bi-Lipschitz equivalent to $\mathbb{Z}^{d}$.

Notice that Theorem 32 is trivial when the dimension $d=1$ since, in this case, every Delone set (with no extra assumptions) is bi-Lipschitz equivalent to $\mathbb{Z}$. As an application of the work of Laczkovich $[\mathrm{L}]$, Solomon in Solo showed also that for every self-similar tiling of $\mathbb{R}^{d}$ of Pisot type there is a bounded displacement between its associated Delone set $X$ and $\beta \mathbb{Z}^{d}$ for a $\beta>0$ (i.e. there is a bijection $\phi: X \rightarrow \beta \mathbb{Z}^{d}$ such that $\Phi-I d$ is bounded).

The strategy of the proof of Theorem 32 is the following. First consider the easy case where all the Voronoï cells $V$ of a Delone set $X$ have an unit volume. Thus any finite union of $N$ Voronoï cells meet at least $N$ unit squares, 
and conversely $N$ unit squares meet at least $N$ Voronoï cells. So by the transfinite form of Hall's marriage Lemma, there exists a bijection between the collection of Vornoï cells and the units squares, so that any cell intersects its image. This define a map $\phi: X \rightarrow \mathbb{Z}^{d}$ such that $\phi-I d$ is bounded.

For the general case, we need to consider the measurable function $f: \mathbb{R}^{d} \rightarrow \mathbb{R}$ defined by

$$
f(x)=\sum_{y: x \in V_{y}} \frac{1}{\operatorname{vol} V_{y}} \quad x \in \mathbb{R}^{d},
$$

where $V_{y}$ denotes the Voronoï cell of the point $y \in X$. If $\phi: \mathbb{R}^{d} \rightarrow \mathbb{R}^{d}$ is a bi-Lipschitz map so that its Jacobian determinant is $f$, standard calculus show us that the image $\phi(V)$ of any Voronoï cell $V$ of $X$ has volume 1 . The proof of Theorem 32 consists then to generalize to all dimension $d$ a sufficient condition given by Burago and Kleiner BK1 in dimension 2 to solve the equation det $\mathrm{D} \phi=f$ with $\phi$ an unknown bi-Lipschitz map. This condition involves analytical tools and the density deviation of the points of $X$ with respect to its average. This last point is controlled by the Lagarias and Pleasants Theorem 16 .

\section{References}

[AC] J. Aliste Prieto, D. Coronel. Tower systems for linearly repetitive Delone sets, Ergod. Th. and Dynam. Sys. (2011) 316 pp.1595 -1618

[ACG] J. Aliste Prieto, D. Coronel, J.-M. Gambaudo Rapid convergence to frequency for substitution tilings of the plane. Comm. Math. Phys. 306 (2011), no. $2,365-380$

[ACG1] J. Aliste Prieto, D. Coronel, J.-M. Gambaudo Linearly repetitive Delone sets are rectifiable. arXiv:1103.5423.

[BBG] J. Bellissard, R. Benedetti, J.-M. Gambaudo, Spaces of tilings, finite telescopic approximations and gap-labeling. Comm. Math. Phys. 261 (2006), no. $1,1-41$.

[BG] R. Benedetti, J.-M. Gambaudo, On the dynamics of $\mathbb{G}$-solenoids. Applications to Delone sets. Ergodic Theory Dynam. Systems 23 (2003), no. 3, 673-691

[Bes] A. Besbes, Contributions a l'étude de quelques systèmes quasicrystallographics, Ph.D. thesis, Université Pierre et Marie Curie, Paris, 2008.

[BBL] A. Besbes, M. Boshernitzan, D. Lenz, Delone sets with finite local complexity: linear repetitivity versus positivity of weights. preprint

[Bo] M. Boshernitzan, A condition for unique ergodicity of minimal symbolic flows. Ergodic Theory Dynam. Systems 12 (1992), no. 3, 425-428.

[BDM1] X. Bressaud, F. Durand, and A. Maass, Necessary and sufficient conditions to be an eigenvalue for linearly recurrent dynamical Cantor systems, J. London Math. Soc. (2) 72 (2005), no. 3, 799-816.

[BDM2] X. Bressaud, F. Durand, and A.Maass, On the eigenvalues of finite rank Bratteli-Vershik dynamical systems. Ergodic Theory Dynam. Systems 30 (2010), no. 3, 639-664 
[Bru] N. G. de Bruijn, Algebraic theory of Penrose's nonperiodic tilings of the plane. I, II. Nederl. Akad. Wetensch. Indag. Math. 43 (1981), no. 1, 39-52, $53-66$.

[BK] D. Burago, B. Kleiner, Separated nets in Euclidean space and Jacobians of bi-Lipschitz maps. Geom. Funct. Anal. 8 (1998), no. 2, 273-282.

[BK1] D. Burago, B. Kleiner, Rectifying separated nets. Geom. Funct. Anal. 12 (2002), no. 1, 80-92.

[CD] M. I. Cortez, F. Durand, Self-similar tiling systems, topological factors and stretching factors, Discrete Comput. Geom. 40 (2008), no. 4, 622-640.

[CDHM] M. I. Cortez, F. Durand, B. Host, A. Maass, Continuous and measurable eigenfunctions of linearly recurrent dynamical Cantor systems. J. London Math. Soc. (2) 67 (2003), no. 3, 790-804.

[CDP] M. I. Cortez, F. Durand, S. Petite, Linearly repetitive Delone systems have a finite number of nonperiodic Delone system factors. Proc. Amer. Math. Soc. 138 (2010), no. 3, 1033-1046.

[CD] M. I. Cortez, F. Durand, Self-similar tiling systems, topological factors and stretching factors. Discrete Comput. Geom. 40 (2008), no. 4, 622-640.

[CGM] M. I. Cortez, J. M. Gambaudo, and A. Maass, Rotation topological factors of minimal $\mathbb{Z}^{d}$-actions of the Cantor set, Trans. Amer. Math. Soc. 359 (2007), no. 5, 2305-2315.

[C] D. Coronel, The cohomological equation over dynamical systems arising from Delone sets. Ergod. Th. and Dynam. Sys. 31 (2011), no. 3, 807-833

[C0] D. Coronel, Ecuaciones cohomológicas sobre espacios de embaldosados, Ph.D. thesis, Univ. de Chile, Univ. de Nice-Sophia Antipolis, Santiago, Nice, 2009.

[DL] D. Damanik, D. Lenz, Linear repetitivity I., Uniform subadditive ergodic theorems and applications, Discrete and Computational Geometry, 26 (2001), 411-428.

[DK] F.M. Dekking, M. Keane, Mixing properties of substitutions, Zeit. Wahr. 42 (1978), 23-33

[Du] F. Durand, Contributions à l'étude des suites et systèmes dynamiques substitutifs, Ph.D. thesis, Univ. de la Méditerrannée, Aix-Marseille II, Marseille, 1996.

[Du0] F. Durand, A characterization of substitutive sequences using return words, Discrete Mathematics 179 (1998), 89-101.

[Du1] F. Durand, Linearly recurrent subshifts have a finite number of non-periodic subshift factors, Ergod. Th. and Dynam. Sys. 20 (2000), 1061-1078.

[Du2] F. Durand, Corrigendum and addendum to: Linearly recurrent subshifts have a finite number of non-periodic subshift factors, Ergod. Th. and Dynam. Sys. 23 (2003), 663-669.

[DHS] F. Durand, B. Host, C. Skau, Substitutional dynamical systems, Bratteli diagrams and dimension groups, Ergod. Th. and Dynam. Sys. 19 (1999), 953-993.

[Gh] Étienne Ghys, Laminations par surfaces de Riemann, Dynamique et géométrie complexes (Lyon, 1997), vol. 8, Soc. Math. France, Paris, 1999, pp. -49 . 
[Gro93] M. Gromov, Asymptotic invariants of infinite groups. Geometric group theory, Vol. 2 (Sussex, 1991), 1-295, London Math. Soc. Lecture Note Ser., 182, Cambridge Univ. Press, Cambridge, 1993.

[H] Host, B. Valeurs propres des systèmes dynamiques définis par des substitutions de longueur variable. Ergodic Theory Dynam. Systems 6 (1986), no. $4,529-540$.

[HRS] C. Holton, C. Radin, L. Sadun, Conjugacies for tiling dynamical systems, Comm. Math. Phys. 254 (2005), 343-359.

[L] M. Laczkovich, Uniformly spread discrete sets in $\mathbb{R}^{d} d$, Journal of the London Mathematical Society. Second Series 46 (1992), 39-57.

[LP1] J. C. Lagarias, P. A. B. Pleasants, Local complexity of Delone sets and crystallinity. Dedicated to Robert V. Moody. Canad. Math. Bull. 45 (2002), no. 4, 634-652

[LP2] J. C. Lagarias, P. A. B. Pleasants, Repetitive Delone sets and quasicrystals, Ergod. Th. and Dynam. Sys. 23 (2003), 831-867.

[Le02] D. Lenz, Uniform ergodic theorems on subshifts over a finite alphabet. Ergodic Theory Dynam. Systems 22 (2002), no. 1, 245-255.

[Le] D. Lenz, Aperiodic linearly repetitive Delone sets are densely repetitive. Discrete Comput. Geom. 31 (2004), no. 2, 323-326

[McM] C.T. McMullen, Lipschitz maps and nets in Euclidean space. Geom. Funct. Anal. 8 (1998), 304-314.

[MH] M. Morse, G. Hedlund, Symbolic dynamics II. Sturmian trajectories. Amer. J. Math. 62, (1940). 1-42.

[Pe] K. Petersen, Factor maps between tiling dynamical systems, Forum Math. 11 (1999), 503-512.

[RS] C. Radin, L. Sadun, Isomorphism of hierarchical structures, Ergod. Th. and Dynam. Sys. 21 (2001), 1239-1248.

[Solo] Y. Solomon, Substitution Tilings and Separated Nets with Similarities to the Integer Lattice, Israel J. Math. 181 (2011), no. 1, 445-460.

[So1] B. Solomyak, Dynamics of self-similar tilings, Ergod. Th. and Dynam. Sys. 17 (1997), 695-738.

[So2] B. Solomyak, Nonperiodicity implies unique composition for self-similar translationally finite tilings, Discrete Comput. Geom. 20 (1998), 265-279.

[So3] B. Solomyak, Spectrum of dynamical systems arising from Delone sets, In: Quasicrystals and discrete geometry (Toronto, ON, 1995), Providence, RI: Am. Math. Soc.,1998, 265-275.

[Tol] D. Toledo, Geometric group theory, 2: Asymptotic invariants of finite groups by M. Gromov. Bull. Amer. Math. Soc. 33 (1996), 395-398.

José Aliste-Prieto, Daniel Coronel

\author{
María Isabel Cortez \\ Departamento de Matemática y CC. de la Universidad de Santiago de Chile, Av. \\ Libertador Bernardo O'Higgins 3363. \\ e-mail: mcortez@usach.cl
}


Fabien Durand

Laboratoire Amiénois de Mathématique Fondamentale et Appliquée, CNRS-UMR 7352, Université de Picardie Jules Verne, 33 rue Saint Leu, 80039 Amiens Cedex, France.

e-mail: fabien.durand@u-picardie.fr

Samuel Petite

e-mail: samuel.petite@u-picardie.fr 